\title{
Chern-Simons states at genus one
}

\author{
Fernando Falceto円 \\ Krzysztof Gawȩdzki \\ C.N.R.S., I.H.E.S., 91440 Bures-sur-Yvette, France
}

\begin{abstract}
We present a rigorous analysis of the Schrödinger picture quantization for the $S U(2)$ Chern-Simons theory on 3-manifold torus $\times$ line, with insertions of Wilson lines. The quantum states, defined as gauge covariant holomorphic functionals of smooth $s u(2)$ connections on the torus, are expressed by degree $2 k$ theta-functions satisfying additional conditions. The conditions are obtained by splitting the space of semistable $s u(2)-$ connections into nine submanifolds and by analyzing the behavior of states at four codimension 1 strata. We construct the Knizhnik-Zamolodchikov-Bernard connection allowing to compare the states for different complex structures of the torus and different positions of the Wilson lines. By letting two Wilson lines come together, we prove a recursion relation for the dimensions of the spaces of states which, together with the (unproven) absence of states for spins $>\frac{1}{2}$ level implies the Verlinde dimension formula.
\end{abstract}

\section{Introduction}

Since the Chern-Simons (CS) theory was revisited by E. Witten in [33], with the stress on its topological nature, a considerable effort has been made to study different aspects of the theory. From the point of view of covariant quantization, the CS model was used to obtain 3-manifold, knot and link invariants, either by surgery [33] [22] or in the perturbative expansion [7] [18] [3].

Here we will discuss a complementary aspect of the CS theory: its canonical quantization. It has been argued in 33 that the space of Schrödinger states of the CS theory with a compact Lie group $G$, in the presence of Wilson lines, is isomorphic to the space of conformal blocks of the associated group $G$ Wess-Zumino-Witten (WZW) conformal field theory. The Wilson lines correspond to the insertions of the primary fields in the two-dimensional model. The conformal blocks are holomorphic sections of the Friedan-Shenker (FS) 13 vector bundle over the moduli space of complex structures of a punctured Riemann surface. By definition, they are horizontal

\footnotetext{
${ }^{1}$ address after October 1, 1992: Depto. Física Teórica, U. Zaragoza, 50009 Zaragoza, Spain
} 
with respect to a flat projective connection. In the WZW model, the latter is a generalization [4] [5] [33] [21] [16] [2] of the genus zero Knizhnik-Zamolodchikov (KZ) connection [24] 23].

For the case with no Wilson lines, the argument of [33] goes like follows. Working on a 3-manifold of the form $\Sigma \times \mathbb{R}$, with $\Sigma$ a 2 -surface and $\mathbb{R}$ interpreted as time, one identifies the phase space of the Chern-Simons theory as the moduli space of flat $G$-connections on $\Sigma$. The choice of a complex structure on $\Sigma$ allows to replace the latter with the moduli space of holomorphic $G^{\mathbb{C}}$-bundles on $\Sigma[27[$ [ ]. It determines, in the geometric quantization jargon, a complex polarization of the phase space and, consequently, a concrete realization of the space of CS states: they become holomorphic sections of a power $\sim$ to the coupling constant (level) $k$ of the determinant line bundle over the moduli space of $G^{\mathbb{C}}$-bundles. Such sections form the fiber of the FS bundle for the WZW model with the point in the base given by the complex structure of $\Sigma$. The KZ connection in the FS bundle allows to compare the CS states for different polarizations.

The presence of the Wilson lines parallel to the time axis, carrying representations $R_{n}$ of $G$, leads to a slight modification of the above picture. If we first treat the insertions classically then the flat $G$-connections forming the phase space should have prescribed holonomies (up to conjugacy) around the punctures and, given the complex structure of $\Sigma$, one obtains the moduli space of holomorphic $G^{\mathbb{C}}$-bundles on $\Sigma$ with parabolic structure at the punctures 25$]$. The CS states are holomorphic sections of a power $(\sim k)$ of the determinant line bundle over the new moduli space.

Our approach will be somewhat different from the above. First, one may treat the Wilson lines quantum-mechanically from the start. The space of states which results then is composed of holomorphic sections of a finite-dimensional vector bundle over the old moduli space of $G^{\mathbb{C}}$ bundles on $\Sigma$ (without parabolic structures). The fiber of the bundle is the tensor product of the representation spaces $V_{R_{n}}$, one for each Wilson line.

The two approaches should be equivalent provided the subtle point of the behavior of the sections around singular points of the moduli spaces is treated appropriately (stability, etc.). We shall essentially follow the second approach with another modification which allows precision and avoids at the onset the subtleties inherent in the finite-dimensional moduli-space context at a relatively low cost (one has to work with infinite-dimensional Fréchet spaces). Namely, we shall define the CS states as holomorphic maps from the space of smooth $G$-connections on $\Sigma$ to $\otimes V_{R_{n}}$ with prescribed transformation properties under the complex gauge transformations (gauge covariant). This infinite-dimensional definition is natural in both the CS setting (where it corresponds to "quantize first, impose gauge conditions next" approach) and in the WZW theory (it produces there the solutions of the current algebra Ward identities). Heuristically, our approach gives sections of a $\otimes V_{R_{n}}$-vector bundle over the space of orbits of $G$-connections under the complex gauge transformations which coincides with the moduli space of holomorphic $G^{\mathbb{C}}$-bundles on $\Sigma$.

The rigorous problem addressed is to describe the spaces of states defined as above, together with the KZ connection, in finite-dimensional terms and as explicitly as possible. Another open (even at genus zero) mathematical problem which, however, we shall not discuss below, is to prove that the $\mathrm{KZ}$ connection is metric [15] [16][10]. E. Verlinde, using the expected factorization properties of the WZW conformal blocks together with their modular properties has given a 
formula for the dimension of the FS bundles [31]2. This formula has been rigorously proven in some special cases and from various starting points ${ }^{3}$.

In [6] a proof for the $S U(2)$ case with no insertions but in general topology was given within the moduli space approach.

Recently, G. Faltings has announced a proof of the general result [11.

In [17], a reduction of the infinite-dimensional formulation to finite-dimensional setup was achieved for $G=S U(2)$ and genus zero. The spaces of spherical CS states were realized as subspaces of $\operatorname{Inv}\left(\otimes V_{R_{n}}\right)$ composed of the $G$-invariant tensors satisfying explicit conditions stable under the $\mathrm{KZ}$ connection. The factorization properties of these spaces implying the Verlinde formula for their dimensions where proven. The present paper is, essentially, an extension of this work to the genus 1 case. We realize the toroidal CS states as theta-functions satisfying explicit conditions. The flat (projective) connection allowing comparing the states for different insertion points and different complex structures on the torus is also constructed. The factorization properties of the spaces of toroidal states when two insertion points coincide allow us to prove the Verlinde formula modulo the assumption that there are no states if one of the inserted spins is larger than $k / 2$. The latter fact was proven in the spherical case in [17]. At genus one, it should follow by the factorization when the torus is pinched to the sphere. We postpone the study of this factorization, more difficult technically, to a future work.

The organization of the paper is as follows. In Section 2 we define precisely the theory and describe our picture of the Chern-Simons states as given by gauge covariant holomorphic functionals on the space of $G$-connections. In Section 3 we describe a stratification of the space of $S U(2)$-connections ( $\cong$ the space of holomorphic $S L(2, \mathbb{C})$-bundles). It is a refinement of the stratification given in [1]. We need a finer decomposition of the semistable stratum of [1] which then provides the main tool in the reduction of the infinite-dimensional formulation to a finite-dimensional one. The reduction associating to every state a set of theta-functions is performed in Section 4. In Section 5, we construct the toroidal version of the KZ connection on the FS bundle with fibers composed of the toroidal CS states and the base given by the moduli space of punctured complex tori. In Section 6, we extend the bundle and the connection to the component of the boundary of the base corresponding to two punctures coming together, show the factorization of the fibers there and prove the Verlinde formula for the dimension of the space of states (all that modulo the assumption that there are no states with spins $>k / 2$ ). Finally in Section 7 we discuss our conclusions and foresee future lines of research. Several appendices collect proofs and properties that are used in the paper.

\section{Chern-Simons theory}

We shall study the CS theory on a three-dimensional manifold $\mathcal{M}=\Sigma \times \mathbb{R}$ where $\Sigma$ is a compact Riemann surface without boundary and $\mathbb{R}$ plays the role of time. The action of the theory at

2 31] contains the formula for the case with up to three insertions on the sphere and for the arbitrary genus but no insertions; its extension to the general case may be found in [26]

${ }^{3}$ see 29]30] for the earlier related work 
level $k \in \mathbb{N}$ and with Lie group $G=S U(n)$ is given by

$$
S=\frac{k}{4 \pi} \int_{\mathcal{M}} \operatorname{tr}\left(B d B+\frac{2}{3} B^{3}\right)
$$

where $B$ is the Yang-Mills connection on the trivial $G$-bundle over $\mathcal{M}$ i.e. an $\mathfrak{s u}(n)$-valued 1-form on $\mathcal{M}$. We shall also consider the insertion of $N$ disjoint Wilson lines $P_{n} \times \mathbb{R}$ carrying irreducible representations $R_{n}$ of $G$.

In the temporal gauge $B_{0}=0$, the phase space of the theory is the set $\mathcal{A}$ of two-dimensional connections $A$ on the trivial $G$-bundle over $\Sigma$ with symplectic form $\frac{k}{4 \pi} \int \operatorname{tr}(\delta A)^{2}$. Due to the remaining gauge freedom, this phase space has to be constrained further by imposing an appropriate flatness condition on connections $A$ [33], [9] (see below).

To quantize the theory, one may use the complex structure on $\mathcal{A}$ induced by the complex structure on the Riemann surface: the splitting $\mathcal{A} \ni A=A_{\bar{z}} d \bar{z}+A_{z} d z$ allows to identify $\mathcal{A}$ with the complex space $\mathcal{A}^{01}$ of $\mathfrak{s l}(n, \mathbb{C})$-valued $(0,1)$-forms on $\Sigma$. The quantum states in the holomorphic quantization à la Bargmann of the theory are given (see [14 [9] 115] 16 17]) by holomorphic functionals $\Psi$ of $A^{01}=A_{\bar{z}} d \bar{z} \in \mathcal{A}^{01}$ with values in $\otimes_{n=1}^{N} V_{R_{n}}$ where $V_{R}$ denotes the vector space of representation $R$. States $\Psi$ must also satisfy the quantum flatness condition

$$
\left(F^{a}(z)-\sum_{n=1}^{N} \frac{2 \pi}{k} \delta\left(z-z_{n}\right) t_{(n)}^{a}\right) \Psi\left(A^{01}\right)=0
$$

where generators $t^{a}$ of $\mathfrak{s u}(n)$ are normalized so that $\operatorname{tr}\left(t^{a} t^{b}\right)=\frac{1}{2} \delta^{a b}, t_{(n)}^{a}$ stands for the action of $t^{a}$ on the $n$-th factor of $\otimes_{n} V_{R_{n}}$ and

$$
F=\sum_{a} F^{a} t^{a}=\partial_{z} A_{\bar{z}}+\partial_{\bar{z}} \frac{\pi}{k} \frac{\delta}{\delta A_{\bar{z}}}-\left[\frac{\pi}{k} \frac{\delta}{\delta A_{\bar{z}}}, A_{\bar{z}}\right]
$$

is the curvature of $A=\sum_{a} A^{a} t^{a}$ with $A_{z}^{a}$ replaced by its quantum version $-\frac{\pi}{k} \frac{\delta}{\delta A_{\bar{z}}^{a}}$.

To introduce holomorphic functionals we need a differentiable structure in the space $\mathcal{A}^{01}$ of smooth $(0,1)$-connections. We shall consider this space with the $C^{\infty}$ topology. By definition, holomorphic functionals on it are $C^{\infty}$-smooth in the Fréchet sense 20] with the complex-linear derivative.

The flatness condition (2.2) may be integrated in the following sense. Let $\mathcal{G}^{\mathbb{C}}$ denote the space of complex gauge transformations i.e. of smooth maps $h: \Sigma \rightarrow S L(n, \mathbb{C})$ which act on $\mathcal{A}^{01}$ by

$$
A^{01} \mapsto{ }^{h} A^{01} \equiv h A^{01} h^{-1}+h \bar{\partial} h^{-1}
$$

with $\bar{\partial}=d \bar{z} \partial_{\bar{z}}$. Then (2.2) is equivalent to demanding that

$$
\Psi\left({ }^{h} A^{01}\right)=\mathrm{e}^{k S_{W Z W}\left(h^{-1}, A^{01}\right)} \prod_{n} h\left(z_{n}\right)_{(n)} \Psi\left(A^{01}\right),
$$


for every $h \in \mathcal{G}^{\mathbb{C}}$. $S_{W Z W}\left(h, A^{01}\right)$ is the action of the Wess-Zumino-Witten (WZW) model [32] coupled to the $(0,1)$-component of the gauge field. Explicitly,

$$
S_{W Z W}\left(h, A^{01}\right)=-\frac{i}{4 \pi} \int_{\Sigma} \operatorname{tr}\left(h^{-1} \partial h\right)\left(h^{-1} \bar{\partial} h\right)-\frac{i}{12 \pi} \int_{\Sigma} d^{-1} \operatorname{tr}\left(h^{-1} d h\right)^{3}+\frac{i}{2 \pi} \int_{\Sigma} \operatorname{tr}\left(h \partial h^{-1}\right) A^{01}
$$

and is defined modulo $2 \pi i$. The cocycle property of the action

$$
S_{W Z W}\left(h h^{\prime},{ }^{h} A^{01}\right)=S_{W Z W}\left(h^{\prime}, A^{01}\right)-S_{W Z W}\left(h^{-1}, A^{01}\right)
$$

makes (2.5) consistent under the product of gauge transformations.

If we formally define WZW Green functions by the functional integral

$$
\left\langle\Phi_{1}, \ldots, \Phi_{N}\right\rangle_{1, A^{01}}=\int \otimes_{n} g\left(z_{n}\right)_{(n)} \mathrm{e}^{-k S_{W Z W}\left(g, A^{01}\right)} \prod_{z} d g(z)
$$

(here $g_{(n)}$ stands for the matrix representing $g$ in representation $R_{n}$ ) then, from the transformation of the action, one derives the Ward identity

$$
\left\langle\Phi_{1}, \ldots, \Phi_{N}\right\rangle_{1, h_{A}^{01}}=\mathrm{e}^{k S_{W Z W}\left(h^{-1}, A^{01}\right)} \prod_{n} h\left(z_{n}\right)_{(n)}\left\langle\Phi_{1}, \ldots, \Phi_{N}\right\rangle_{1, A^{01}}
$$

which makes clear the relation between the space of states of the Chern-Simons theory and the solutions of the chiral Ward identity (2.9) of the WZW model.

From eq. (2.5) we see that $\Psi$ will be determined once we know its value at a point of each $\mathcal{G}^{\mathbb{C}}$ orbit. However space $\mathcal{A}^{01} / \mathcal{G}^{\mathbb{C}}$ is, in general, not a manifold and the smoothness of $\Psi$ is not given for free. In the following section we shall study the relative positions and codimensions of the $\mathcal{G}^{\mathbb{C}}$ orbits as an introductory step to finding the holomorphic functionals $\Psi$ with transformation rule (2.5).

\section{Stratification of the space of connections}

In this section we shall describe a stratification of $\mathcal{A}^{01}$ into submanifolds of finite codimension invariant under the action of the gauge group.

First note that the space $\mathcal{A}^{01}$ may be identified with the space of holomorphic $S L(n, \mathbb{C})$ bundles (more exactly, of the structures of a holomorphic $S L(n, \mathbb{C}$ ) vector bundle in the trivial bundle $\left.\Sigma \times \mathbb{C}^{n}\right)$. For $A^{01} \in \mathcal{A}^{01}, \bar{\partial}+A^{01}$ is the holomorphic derivative of the sections of the corresponding holomorphic bundle. Isomorphic bundles correspond to gauge-related forms so that the space of orbits $\mathcal{A}^{01} / \mathcal{G}^{\mathbb{C}}$ may be identified with the space of isomorphism classes of holomorphic $S L(n, \mathbb{C})$ vector bundles. Below, we shall use the bundle language whenever it is more convenient.

We recall from [1] some definitions and results about the holomorphic vector bundles. Let $E$ be such a bundle, $n(E)$ its rank and $k(E)$ its Chern class $(k(E)=0$ if the bundle has structure 
group $S L(n, \mathbb{C}))$. The ratio $\mu(E)=k(E) / n(E)$ is called the slope of $E$. $E$ is called semistable (stable) if for every proper holomorphic vector subbundle $D \subset E, \mu(D) \leq \mu(E)(\mu(D)<\mu(E))$. Every holomorphic bundle $E$ has a canonical filtration

$$
0=E_{0} \subset E_{1} \subset \cdots \subset E_{r}=E
$$

such that $D_{i}=E_{i} / E_{i-1}$ is semistable and

$$
\mu\left(D_{i}\right)<\mu\left(D_{i-1}\right), i=1, \cdots, r
$$

The ranks $n_{i}=n\left(D_{i}\right)$ and Chern classes $k_{i}=k\left(D_{i}\right)$, subject to $\sum_{i} n_{i}=n(E)=n, \sum_{i} k_{i}=0$ and (3.1), determine the stability type of $E$. Of course, if $E$ is semistable, $r=1$.

With the use of these data, $\mathcal{A}^{01}$ is decomposed into submanifolds $\mathcal{A}_{\lambda}^{01}$ formed by all bundles of type $\lambda=\left(\left(n_{i}, k_{i}\right), i=1, \cdots, r\right)$. As the type of a bundle is canonical, $\mathcal{A}_{\lambda}^{01}$ is a union of $\mathcal{G}^{\mathbb{C}}$-orbits (isomorphism classes). If $\mathcal{L}$ denotes the set of all possible types $\lambda$ then

$$
\mathcal{A}^{01}=\bigcup_{\lambda \in \mathcal{L}} \mathcal{A}_{\lambda}^{01}
$$

One may partially order $\mathcal{L}$ by setting $\lambda \preceq \mu$ if $\mathcal{P}(\lambda) \subset \mathcal{P}(\mu)$ where $\mathcal{P}(\lambda)$ is the region in the twodimensional plane between the horizontal axis and the convex polygon whose edges are vectors $\left(n_{i}, k_{i}\right)$ taken in order. The decomposition (stratification) (3.2) has the following property [1]:

$$
\text { For every } I \subset \mathcal{L}, \quad \mathcal{S}_{I}=\bigcup_{\mu \in I} \bigcup_{\lambda \preceq \mu} \mathcal{A}_{\lambda}^{01} \text { is an open submanifold of } \mathcal{A}^{01}
$$

The codimension of $\mathcal{A}_{\lambda}^{01}$ is

$$
c_{\lambda}=\sum_{r \geq i>j \geq 0}\left[\left(n_{i} k_{j}-n_{j} k_{i}\right)+n_{i} n_{j}(g-1)\right]
$$

where $g$ is the genus of $\Sigma$. The stratum $\mathcal{A}_{s s}^{01}$ of semistable bundles, associated to the minimal element $\lambda_{s s}=((n, k))$ of $\mathcal{L}$, is the only one of codimension zero and is an open dense subset of $\mathcal{A}^{01}$. For the genus $g=1$ case under consideration in this paper, we shall need to refine the above stratification, preserving still property (3.3). This will be done by decomposing the main stratum $\mathcal{A}_{s s}^{01}$ into other strata.

From the Weil theorem [19], we know that each isomorphism class of semistable holomorphic vector bundles of vanishing Chern class (on a general Riemann surface) has a flat representative (for $g=1$ also the converse is true). In other words, for each semistable $A^{01}$ there exists an $\mathfrak{s l}(n, \mathbb{C})$-valued $(1,0)$-form $A^{10}$ such that $A=A^{10}+A^{01}$ is flat i.e. $F(A)=d A+A \wedge A=0$. To each of these bundles (and to the choice of $A^{10}$ ) there corresponds a homomorphism

$$
\rho: \pi_{1}(\Sigma) \longrightarrow S L(n, \mathbb{C})
$$


given by the holonomy of the flat connection $A$ :

$$
A=h d h^{-1}
$$

where the $S L(n, \mathbb{C})$-valued map $h$ is defined on the universal covering of $\Sigma$ and

$$
h(a \xi)^{-1}=\rho(a) h(\xi)^{-1} \text { for } a \in \pi_{1}(\Sigma) .
$$

If the homomorhisms $\rho$ are related by conjugation, then they come from isomorphic bundles (since the corresponding flat connections are related by an $S L(n, \mathbb{C})$-valued gauge transformation). In the toroidal case, i.e. when $\Sigma=\mathbb{C} /(2 \pi \mathbb{Z}+2 \pi \tau \mathbb{Z})$ for $\tau \equiv \tau_{1}+i \tau_{2}, \tau_{2}>0$, we have a specially simple situation as the fundamental group of $\Sigma$ is generated by two commuting elements. Then the representations $\rho$ of the fundamental group are given by pairs of commuting matrices $\rho_{1}, \rho_{\tau} \in S L(n, \mathbb{C})$.

For the rank $n=2$ to which we shall limit ourselves from now on, we can distinguish three classes of orbits of equivalent semistable bundles according to the behavior of $\rho$ for (one of) their flat representatives

Case 1. One of the matrices is diagonalizable, and different from $\pm I$, then both are simultaneously diagonalizable.

In this case, we may diagonalize by conjugation both matrices and take:

$$
\rho_{\mu}=\left(\begin{array}{cc}
e^{\lambda_{\mu}} & 0 \\
0 & e^{-\lambda_{\mu}}
\end{array}\right) \equiv e^{\lambda_{\mu} \sigma_{3}}, \quad \mu=1, \tau .
$$

We may still use the freedom of choice of the flat representative for given bundle by replacing the map $h$ of (3.5) by $h \widetilde{h}$ where

$$
\widetilde{h}=e^{(2 \pi)^{-1} z \lambda_{1} \sigma_{3}} .
$$

This does not change $A^{01}$ but changes the transition matrices to

$$
\rho_{1}=I, \quad \rho_{\tau}=e^{2 \pi i u \sigma_{3}}
$$

where $2 \pi i u=\lambda_{\tau}-\tau \lambda_{1}$. One can take

$$
A_{u}=A_{u}^{10}+A_{u}^{01}=u \sigma_{3}(d z-d \bar{z}) / 2 \tau_{2}
$$

as the associated flat connection.

There are gauge relations between chiral connections $A_{u}^{01}$. Let

$$
h_{v}=\exp \left[\frac{v \bar{z}-\bar{v} z}{2 \tau_{2}} \sigma_{3}\right]
$$

be a map from the complex plane to $S L(2, \mathbb{C})$. For $v=m+\tau n \in \mathbb{Z}+\tau \mathbb{Z}, h_{v}$ defines a gauge transformation on the torus which shifts $u$ of $A_{u}^{01}$ by $m+\tau n$. Also, the constant gauge transformations with values in the normalizer of the Cartan subgroup map $A_{u}^{01}$ to itself or to 
$A_{-u}^{01}$. A direct check shows that for $u \notin(\mathbb{Z}+\tau \mathbb{Z}) / 2$ these generate the only gauge transformations relating $A_{u}^{01}$ 's. To generate all the gauge transformations relating $A_{u=0}^{01}$ to other $A_{u}^{01}$ 's, one has to add the arbitrary constant gauge automorphisms $g_{0}$ of $A_{0}^{01}$ to $h_{v}$ 's with $v \in \mathbb{Z}+\tau \mathbb{Z}$. To understand the gauge relations of an arbitrary $A_{u}^{01}, u \in(\mathbb{Z}+\tau \mathbb{Z}) / 2$, to other $A_{u}^{01}$ 's, notice that for $v \in(\mathbb{Z}+\tau \mathbb{Z}) / 2$, although $h_{v} \notin \mathcal{G}^{\mathbb{C}}$ if $v \notin \mathbb{Z}+\tau \mathbb{Z}$ as it is multivalued on the torus, it still defines an automorphism $A^{01} \mapsto{ }^{h_{v}} A^{01}$ of $\mathcal{A}^{01}$ since $h_{v}$ is multiplied by $\pm I$ when one goes around the torus' cycles. The action of such $h_{v}$ still shifts $u$ of $A_{u}^{01}$ by $v \in(\mathbb{Z}+\tau \mathbb{Z}) / 2$ and we may use it to infer all gauge automorphism connecting any $A_{u}^{01}$ with $u \in(\mathbb{Z}+\tau \mathbb{Z}) / 2$ to any other $A_{u}$ 's. They are generated by the automorphisms $h_{u} g_{0} h_{u}^{-1}$ of $A_{u}^{01}(u \in(\mathbb{Z}+\tau \mathbb{Z}) / 2)$ and $h_{v}$ 's with $v \in \mathbb{Z}+\tau \mathbb{Z}$.

It follows that we may split the union of $\mathcal{G}^{\mathbb{C}}$-orbits of chiral connections $A_{u}^{01}$ into five disjoint sets. $\mathcal{A}_{0}^{01}$ will contain the orbits of $A_{u}^{01}$ with $u \notin(\mathbb{Z}+\tau \mathbb{Z}) / 2$. The orbits of $A_{u}^{01}$ 's with $u \in \alpha+\mathbb{Z}+\tau \mathbb{Z}, \alpha=0,1 / 2, \tau / 2,(1+\tau) / 2$ will form the sets $\mathcal{A}_{(\alpha, 1)}^{01}$.

Case 2. One of the matrices is not diagonalizable.

Then we can always, by conjugation, reduce the two matrices to the form:

$$
\rho_{\mu}= \pm\left(\begin{array}{cc}
1 & a_{\mu} \\
0 & 1
\end{array}\right) \equiv \pm e^{a_{\mu} \sigma_{+}}, \quad \mu=1, \tau
$$

If $a_{\tau} \neq \tau a_{1}$ then using

$$
\widetilde{h}=e^{(2 \pi)^{-1} z a_{1} \sigma_{+}}
$$

as in Case 1 and with a further conjugation, we may equivalently obtain

$$
\rho_{1}= \pm I, \quad \rho_{\tau}= \pm\left(\begin{array}{cc}
1 & 2 \pi i \\
0 & 1
\end{array}\right)
$$

where all four possibilities of combinations of signs \pm are allowed. As the corresponding flat connections we may take

$$
\widehat{A}_{\alpha}=h_{\alpha}\left(\sigma_{+}(d z-d \bar{z}) /\left(2 \tau_{2}\right)\right) h_{\alpha}^{-1}+h_{\alpha} d h_{\alpha}^{-1}
$$

where, for $\alpha=0,1 / 2, \tau / 2,(1+\tau) / 2$ corresponding to signs $(+,+),(+,-),(-,+),(-,-), h_{\alpha}$ are given by (3.8).

The orbits of

$$
\widehat{A}_{\alpha}^{01}=-\left(\alpha \sigma_{3}+e^{\frac{\alpha \bar{z}-\bar{\alpha} z}{\tau_{2}}} \sigma_{+}\right) d \bar{z} /\left(2 \tau_{2}\right)
$$

will form the subsets $\mathcal{A}_{(\alpha, 0)}^{01}$ of $\mathcal{A}^{01}$. Bundles with $a_{\tau}=\tau a_{1}$, excluded from $\mathcal{A}_{(\alpha, 0)}^{01}$, are equivalent to those with transition functions $\pm I$, and they are elements of the sets $\mathcal{A}_{(\alpha, 1)}^{01}$ considered above.

From the remark that the equivalence of holonomies $\rho$ implies that $A^{01}$ 's are in the same orbit it should be clear that the sets $\mathcal{A}_{0}^{01}, \mathcal{A}_{(\alpha, i)}^{01}$ cover the semistable stratum $\mathcal{A}_{s s}^{01}$ (they produce all possible nonequivalent holonomies). It may be less clear that they are all disjoint since they were singled out according to the properties of their (non-unique) flat representatives. Notice 
however that if $A=A^{10}+A^{01}$ and $A^{\prime}=A^{10}+A^{01}$ are two flat representatives of the same $A^{01}$ then $\omega=A^{10}-A^{\prime 10}$ satisfies

$$
\bar{\partial} \omega+A^{01} \wedge \omega+\omega \wedge A^{01}=0 .
$$

A direct check shows that for $A^{01}=\widehat{A}_{\alpha}^{01}$ the solutions are of the form $\omega_{\alpha}=h_{\alpha}\left(a \sigma_{+} d z\right) h_{\alpha}^{-1}$ with complex $a$ and all lead to the same behavior of the holonomy matrices. This demonstrates that sets $\mathcal{A}_{(\alpha, 0)}^{01}$ are mutually disjoint and disjoint from the union of orbits of $A_{u}^{01}$ 's forming (disjoint) sets $\mathcal{A}_{0}^{01}$ and $\mathcal{A}_{(\alpha, 1)}^{01}$.

Now we may assert the main result of this section:

Take the set of indices $\widehat{\mathcal{L}}=\left(\mathcal{L} \backslash\left\{\lambda_{s s}\right\}\right) \cup\{0\} \cup\{(\alpha, 0),(\alpha, 1) \mid \alpha=0,1 / 2, \tau / 2,(1+\tau) / 2\}$. Extend the order relation defined in $\mathcal{L}$ to $\widehat{\mathcal{L}}$ by

$$
0 \preceq(\alpha, 0) \preceq(\alpha, 1) \preceq \lambda \quad \forall \lambda \in \mathcal{L} \backslash\left\{\lambda_{s s}\right\} \text { and } \alpha=0,1 / 2, \tau / 2,(1+\tau) / 2 .
$$

Then:

a) $\mathcal{A}_{\widehat{\lambda}}^{01}$ for $\widehat{\lambda} \in \widehat{\mathcal{L}}$ are connected, mutually disjoint submanifolds of $\mathcal{A}^{01}$. They are invariant under the action of $\mathcal{G}^{\mathbb{C}} \cdot \mathcal{A}^{01}=\bigcup_{\widehat{\lambda} \in \widehat{\mathcal{L}}} \mathcal{A}_{\widehat{\lambda}}^{01}$.

b) For every $\widehat{I} \subset \widehat{\mathcal{L}}$

$$
\mathcal{S}_{\widehat{I}}=\bigcup_{\widehat{\mu} \in \widehat{I} \widehat{\lambda} \preceq \widehat{\mu}} \mathcal{A}_{\widehat{\lambda}}^{01} \quad \text { is an open submanifold of } \mathcal{A}^{01} \text {. }
$$

Consequently, $\mathcal{A}_{\widehat{\lambda}}^{01}$ is a closed submanifold of $\mathcal{S}_{\widehat{I} \cup\{\widehat{\lambda}\}}$ for any minimal element $\widehat{\lambda}$ of $\widehat{\mathcal{L}} \backslash \widehat{I}$.

c) Codimensions $c_{\widehat{\lambda}}$ of $\mathcal{A}_{\widehat{\lambda}}^{01}$ are: $c_{0}=0\left(\mathcal{A}_{0}^{01}\right.$ is an open dense subset of $\left.\mathcal{A}^{01}\right), c_{(\alpha, 0)}=1, c_{(\alpha, 1)}=3$ and $c_{\widehat{\lambda}}$ for $\hat{\lambda} \in \mathcal{L} \backslash\left\{\lambda_{s s}\right\}$ is as in (3.4).

\section{Proof.}

Let $\mathcal{G}_{0}^{\mathbb{C}}$ be the group of gauge transformations which are the identity at the origin and $\mathcal{C} \subset \mathcal{A}^{01}$ the set of constant connections. The elements of the latter may be written as

$$
A_{M}^{01}=-\frac{1}{2} \tau_{2}^{-1} M d \bar{z}, \quad M \in \mathfrak{s l}(2, \mathbb{C}) .
$$

Fix a real number $0<\epsilon<1 / 4$ and take the open set $U_{0} \subset \mathcal{C}$ of constant connections associated to matrices $M \in \mathfrak{s l}(2, \mathbb{C})$ such that

$$
\operatorname{det} M=(a+b \tau)^{2} \quad \text { with } \quad a, b \in(-1 / 2+\epsilon, 1 / 2-\epsilon) \subset \mathbb{R} .
$$

Consider the map

$$
P_{0}: \mathcal{G}_{0}^{\mathbb{C}} \times U_{0} \longrightarrow \mathcal{A}_{s s}^{01}
$$




$$
\left(g, A_{M}^{01}\right) \longmapsto{ }^{g} A_{M}^{01} .
$$

It follows then by the Nash-Moser (inverse function) Theorem [20] that $P_{0}$ is a smooth diffeomorphism onto its (open) image, see Appendix A.

Now take

$$
\begin{aligned}
& V_{0}=\left\{A_{M}^{01} \in U_{0} \mid \operatorname{det}(M) \neq 0\right\}, \\
& V_{(0,0)}=\left\{A_{M}^{01} \in U_{0} \mid \operatorname{det}(M)=0 \text { and } M \neq 0\right\}, \\
& V_{(0,1)}=\{0\} \subset U_{0} .
\end{aligned}
$$

Then $\mathcal{A}_{(0, i)}^{01}=P_{0}\left(\mathcal{G}_{0}^{\mathbb{C}} \times V_{(0, i)}\right)$ and $P_{0}\left(V_{0}\right)$ is an open subset of $\mathcal{A}_{0}^{01}$. It follows that $\mathcal{A}_{(0,0)}^{01}$ and $\mathcal{A}_{(0,1)}^{01}$ have codimensions 1 and 3 , respectively.

For the three other values of $\alpha$, instead of constant connections, we take $U_{\alpha}={ }^{h_{\alpha}} U_{0}$ with $h_{\alpha}$ given by (3.8) and

$$
\begin{aligned}
P_{\alpha}: \mathcal{G}_{0}^{\mathbb{C}} \times U_{\alpha} & \longrightarrow \mathcal{A}^{01} \\
\left(g, A^{01}\right) & \longmapsto{ }^{g} A^{01} .
\end{aligned}
$$

It is clear that $P_{\alpha}$ is also a smooth diffeomorphism onto its image. Then, for $V_{\alpha}={ }^{h_{\alpha}} V_{0}$, we have $\mathcal{A}_{0}^{01}=\bigcup_{\alpha} P_{\alpha}\left(\mathcal{G}_{0}^{\mathbb{C}} \times V_{\alpha}\right)$ and so it is an open manifold dense in $\mathcal{A}_{s s}^{01}$ and, consequently, also in $\mathcal{A}^{01}$. On the other hand $\mathcal{A}_{(\alpha, i)}^{01}={ }^{h_{\alpha}} \mathcal{A}_{(0, i)}^{01}, i=0,1$. From this, and from the properties of the initial stratification (3.3), a), b) and c) follow immediately.

\section{Space of states}

With the detailed knowledge of the space $\mathcal{A}^{01}$ of the chiral connections provided by the preceding Section, we are in a position to describe more effectively the Chern-Simons states defined as the holomorphic functionals $\Psi$ on $\mathcal{A}^{01}$ taking values in $\bigotimes_{n} V_{j_{n}}$ ( $j_{n}$ are spins of the Wilson lines) and transforming according to (2.5) under the (complex) gauge transformations. By considering such a state on the one-parameter family of connections $A_{u}^{01}$ of (3.7), we obtain a holomorphic map $\gamma: \mathbb{C} \longrightarrow \bigotimes_{n} V_{j_{n}}$ given by

$$
\Psi\left(A_{u}^{01}\right) \equiv e^{\pi k u^{2} / \tau_{2}} \prod_{n}\left(e^{\sigma_{3}\left(\bar{z}_{n}-z_{n}\right) u /\left(2 \tau_{2}\right)}\right)_{(n)} \gamma(u) .
$$

The gauge transformations by $h_{m+\tau n}$ (see (3.8)) and by elements of the normalizer of the Cartan subgroup give rise to the conditions

$$
\begin{aligned}
& \gamma(u+1)=\gamma(u), \\
& \gamma(u+\tau)=e^{-2 \pi i k(\tau+2 u)} \prod_{n}\left(e^{i z_{n} \sigma_{3}}\right)_{(n)} \gamma(u),
\end{aligned}
$$




$$
\begin{array}{cc}
0 & =\sum_{n}\left(\sigma_{3}\right)_{(n)} \gamma(u) \\
\gamma(-u) & =\prod_{n}(\Omega)_{(n)} \gamma(u)
\end{array}
$$

where $\Omega$ is the generator of the Weyl group of $S L(2, \mathbb{C})$. Conversely, a holomorphic map $\gamma$ on $\mathbb{C}$ satisfying the above conditions defines by (4.1) and (2.5) a unique functional $\Psi$ on the open dense stratum $\mathcal{A}_{0}^{01}$ satisfying there (2.5). We shall see that such $\Psi$ is holomorphic on $\mathcal{A}_{0}^{01}$ but need not extend to the whole of $\mathcal{A}^{01}$ (if it does then the extension is unique and satisfies (2.5) everywhere). We shall determine below the necessary and sufficient conditions for such an extension to exist. The results of the last Section will be crucial here.

In the polynomial realization of the spaces $V_{j}$ of spin $j$ representations of $S L(2, \mathbb{C})$, elements of $V_{j}$ are polynomials of degree at most $2 j$ with the action of the group by

$$
\left(\begin{array}{ll}
a & b \\
c & d
\end{array}\right)^{-1} P(v)=(c v+d)^{2 j} P\left(\frac{a v+b}{c v+d}\right)
$$

In this realization, $\gamma(u)$ is a polynomial in variables $\underline{v} \equiv\left(v_{n}\right)$ associated to spaces $\left(V_{j_{n}}\right)$. Using (4.2, c) we can see that $\gamma(u)$ is homogeneous of degree $J \equiv \sum_{n} j_{n}$ in variables $\underline{v}$. In particular, this implies that $J$ has to be an integer. We may write

$$
\gamma(u)=\sum_{\underline{m}} f_{\underline{m}}(u) \prod_{n} v_{n}^{j_{n}+m_{n}}
$$

where the sum is over the N-tuples $\underline{m} \equiv\left(m_{n}\right)$ such that $0 \leq j_{n}-\left|m_{n}\right| \in \mathbb{Z}$ and $\sum_{n} m_{n}=0$. From $(4.2, \mathrm{~d})$ we infer that

$$
f_{\underline{m}}(u)=(-1)^{J} f_{-\underline{m}}(-u) .
$$

Finally, from $4.2, \mathrm{a}-\mathrm{b})$,

$$
f_{\underline{m}}(u)=\vartheta\left(u-(4 \pi k)^{-1} \sum_{n} z_{n} m_{n}\right)
$$

with $\vartheta$ an $\underline{m}$-dependent theta-function of degree $2 k$ satisfying

$$
\vartheta(u+1)=\vartheta(u), \quad \vartheta(u+\tau)=e^{-2 \pi i k(\tau+2 u)} \vartheta(u) .
$$

There are $2 k$ independent solutions $\vartheta_{p}$ of eqs. (4.5) given by

$$
\vartheta_{p}(u)=\sum_{n=-\infty}^{\infty} q^{(p+2 k n)^{2} /(4 k)} \mathrm{e}^{2 \pi i u(p+2 k n)}
$$

where $p=0,1, \ldots, 2 k-1$ and $q \equiv e^{2 \pi i \tau}$. Consequently, the spaces of analytic maps $\gamma: \mathbb{C} \rightarrow \otimes_{n} V_{n}$ satisfying (4.2) for fixed $\tau$ and $\underline{z} \equiv\left(z_{n}\right), z_{n} \in \mathbb{C}$, are of finite, constant dimension and form a holomorphic vector bundle $W_{\underline{j}}$ over the space $\mathcal{T}_{N}$ of $(\underline{z}, \tau)$ with $z_{n} \neq z_{n^{\prime}} \bmod (2 \pi, 2 \pi \tau)$. 
The above description of states $\Psi$ defined on flat connections $A_{u}^{01}$ has used a choice of complex coordinates $z_{n}$ whereas the class of connections $A_{u}^{01}$ depends only on the complex structure of the torus. To exhibit the geometric character of $\gamma$ 's notice that different choices of $z_{n}$ 's are intertwined by the action of the discrete group $\Gamma_{N}=S L(2, \mathbb{Z}) \ltimes \mathbb{Z}^{2 N}$ where $\left(\left(\begin{array}{ll}a & b \\ c & d\end{array}\right),\left(p_{n}, r_{n}\right)\right) \in$ $\Gamma_{N}$ acts by mapping $(\underline{z}, \tau)$ to $\left(\underline{z}^{\prime}, \tau^{\prime}\right)$ where

$$
\tau^{\prime}=(a \tau+b) /(c \tau+d), \quad z_{n}^{\prime}=\left(z_{n}+2 \pi p_{n}+2 \pi \tau r_{n}\right) /(c \tau+d) .
$$

The action of $\Gamma_{N}$ on bundle $W_{\underline{j}}$ which lifts the action $(\underline{z}, \tau) \mapsto\left(\underline{z}^{\prime}, \tau^{\prime}\right)$ on the base is given by the formula

$$
\gamma^{\prime}\left(u^{\prime}\right)=e^{2 \pi i k c u^{\prime} u} \prod_{n}\left(e^{-i c z_{n}^{\prime} u \sigma_{3}+2 \pi i r_{n} u \sigma_{3}}\right)_{(n)} \gamma(u),
$$

where $u^{\prime}=u /(c \tau+d)$. $\gamma^{\prime}$ 's related by this action describe the same state $\Psi$ for different choices of complex coordinates $z_{n}$ (notice that $A_{u} \equiv-u \sigma_{3} d \bar{z} /\left(2 \tau_{2}\right)=A_{u^{\prime}}^{\prime} \equiv-u^{\prime} \sigma_{3} d \bar{z}^{\prime} /\left(2 \tau_{2}^{\prime}\right)$ ). Dividing bundle $W_{\underline{j}}$ by the action of $\Gamma_{N}$, one obtains a bundle $W_{\underline{j}} / \Gamma_{N}$ over the moduli space $\mathcal{T}_{N} / \Gamma_{N}$ of tori with $\bar{N}$ punctures.

Let us return to discussing which $\gamma$ 's correspond to global states $\Psi$. By explicitly conjugating a constant matrix $M \equiv\left(\begin{array}{cc}M_{0} & M_{1} \\ M_{2} & -M_{0}\end{array}\right) \in \mathfrak{s l}(2, \mathbb{C})$ such that $\operatorname{det}(M) \neq 0$ and $M_{1} \neq 0$ to a diagonal matrix $u \sigma_{3}$ and by using (4.1) and (2.5), we obtain the following expression for $\Psi\left({ }^{h_{\alpha}} A_{M}^{01}\right)$ (see (3.10) and (3.8)):

$$
\begin{gathered}
\prod_{n}\left(e^{M\left(z_{n}-\bar{z}_{n}\right) /\left(2 \tau_{2}\right) h_{\alpha}\left(z_{n}\right)}\right)_{(n)} \Psi\left({ }^{h_{\alpha}} A_{M}^{01}\right)\left(\left(v_{n}\right)\right) \\
=e^{\pi k\left((u+\alpha)^{2}+2\left(M_{0}-u\right) \bar{\alpha}\right) / \tau_{2}}\left(\prod_{n} e^{-(\alpha-\bar{\alpha}) z_{n} j_{n} / \tau_{2}}\left(M_{1}+\left(M_{0}-u\right) v_{n}\right)^{2 j_{n}}\right) \\
\cdot\left(2 M_{1} u\right)^{-J} \gamma(u+\alpha)\left(\left(e^{(\alpha-\bar{\alpha}) z_{n} / \tau_{2}}\left(M_{1}+\left(M_{0}+u\right) v_{n}\right) /\left(M_{1}+\left(M_{0}-u\right) v_{n}\right)\right)\right)
\end{gathered}
$$

where $u= \pm\left(M_{0}^{2}+M_{1} M_{2}\right)^{1 / 2}$ and properties (4.2, a-d) of $\gamma$ insure that the right hand side is independent of the choice of the sign. This rather messy expression becomes much simpler for $M(u)=\left(\begin{array}{cc}u & 2 \\ 0 & -u\end{array}\right):$

$$
\begin{gathered}
\prod_{n}\left(e^{M(u)\left(z_{n}-\bar{z}_{n}\right) /\left(2 \tau_{2}\right)} h_{\alpha}\left(z_{n}\right)\right)_{(n)} \Psi\left({ }^{h_{\alpha}} A_{M(u)}^{01}\right)\left(\left(v_{n}\right)\right) \\
=e^{\pi k(u+\alpha)^{2} / \tau_{2}}\left(\prod_{n} e^{-(\alpha-\bar{\alpha}) z_{n} j_{n} / \tau_{2}}\right) u^{-J} \gamma(u+\alpha)\left(\left(e^{(\alpha-\bar{\alpha}) z_{n} / \tau_{2}}\left(1+u v_{n}\right)\right)\right) .
\end{gathered}
$$

Notice the negative power $u^{-J}$ on the right hand side which is entire in $u$ if and only if

$$
\left.\widehat{D}_{\underline{j}, \alpha}^{L} \gamma \equiv \partial_{u}^{l_{0}} \partial_{v_{1}}^{l_{1}} \cdots \partial_{v_{N}}^{l_{N}} \gamma(u)(\underline{v})\right|_{v_{n}=\exp \left[(\alpha-\bar{\alpha}) z_{n} / \tau_{2}\right]}=0
$$


for every $N+1$-tuple of non negative integers $L \equiv\left(l_{n}\right)$ such that $|L| \equiv \sum_{n=0}^{N} l_{n}<J$.

Conversely, it is not difficult to see that this condition implies that $\Psi\left({ }^{h_{\alpha}} A_{M}^{01}\right)$, as given by (4.8), is analytic on the set of $M$ 's with $M_{1} \neq 0$. The last requirement may be relaxed to $M \neq 0$ by conjugating matrices $M$. Thus condition (4.10) is necessary and sufficient for $\Psi\left({ }^{h \alpha} A_{M}^{01}\right)$ to extend analytically to the codimension 1 stratum of matrices $M \neq 0$ with $\operatorname{det}(M)=0$.

Now using maps $P_{\alpha}$ of (3.14), we infer the holomorphicity of $\Psi$ on the open dense stratum $\mathcal{A}_{0}^{01}$ and see that it extends analytically to the codimension 1 strata $\mathcal{A}_{(\alpha, 0)}^{01}$ if and only if the conditions (4.10) are satisfied. The extension to the other strata of $\mathcal{A}^{01}$, of codimension $>1$, follows then automatically by applying inductively the Hartogs Theorem. This is there that one uses property b) of the stratification of $\mathcal{A}^{01}$ proven in Section 3.

Summarizing: a holomorphic map $\gamma: \mathbb{C} \longrightarrow \bigotimes_{n} V_{j_{n}}$ satisfying conditions (4.2, a-d) defines by relations (4.1) and (2.5) a (global) Chern-Simons state if and only if it satisfies (4.10) for $\alpha=0,1 / 2, \tau / 2,(1+\tau) / 2$; besides, every Chern-Simons state may be uniquely represented this way.

It is not difficult to see that condition (4.10) is preserved by the action of the discrete group $\Gamma_{N}$ (notice that $A_{M\left(u^{\prime}\right)}^{\prime 01}=e^{(c \tau+d)^{1 / 2} \sigma_{3}} A_{M(u)}^{01} e^{-(c \tau+d)^{1 / 2} \sigma_{3}}$ ). If the spaces of solutions of $(4.10)$ are of constant dimension, they form a holomorphic ("fusion rules") subbundle $W_{\underline{j}}^{f r}$ of $W_{\underline{j}}$ equivariant under the action of $\Gamma_{N}$ and consequently projecting to a bundle over the moduli space $\mathcal{T}_{N} / \Gamma_{N}$.

As an illustration, let compute the spaces of Chern-Simons states with zero and one insertions.

\section{States with zero insertions.}

From (4.3) and (4.4) the zero-points states are simply the even theta-functions of degree $2 k$ since for $J=0$ conditions (4.10) play no role. The space of even theta-functions has dimension $k+1$. It is spanned by the Kac-Moody characters $\chi_{k, j}\left(\tau, \mathrm{e}^{2 \pi i u}\right)$ with fixed $\tau$ and $j=0,1 / 2, \ldots, k / 2$.

\section{States with one insertion.}

In this case the states with one insertion of spin $j \in \mathbb{N}$ are

$$
\gamma(u)(v)=\vartheta(u) v^{j}
$$

where $\vartheta$ is a theta-function of degree $2 k$ with

$$
\begin{aligned}
& \vartheta(-u)=(-1)^{j} \vartheta(u), \\
& \left.\partial_{u}^{l} \vartheta(u)\right|_{u=\alpha}=0 \quad \text { for } l<j \text { and } \alpha=0, \frac{1}{2}, \frac{\tau}{2}, \frac{\tau+1}{2} .
\end{aligned}
$$


To study the dimension $\mathcal{D}_{j}$ of the space of solutions of (4.12,a-b) we shall first focus on the case of even spin, $j \in 2 \mathbb{N}$. In that case $(4.12$, a) requires even theta-functions for which:

$$
\mathrm{e}^{-4 \pi i k u \frac{\alpha-\bar{\alpha}}{\tau-\bar{\tau}}} \vartheta(u+\alpha)=\mathrm{e}^{4 \pi i k u \frac{\alpha-\bar{\alpha}}{\tau-\bar{\tau}}} \vartheta(-u+\alpha) \quad \alpha=0, \frac{1}{2}, \frac{\tau}{2}, \frac{\tau+1}{2} .
$$

As a consequence of (4.13), vanishing of $\left.\partial_{u}^{l} \vartheta(u)\right|_{u=\alpha}$ for $l \leq 2 n \in 2 \mathbb{N}$ implies also vanishing of the same expression for $l=2 n+1$. So in (4.12, b), we may consider only even values of $l$. Thus we are left with $2 j$ linear conditions on the $k+1$ dimensional space of even theta-functions and we obtain the lower bound on the dimension of the space of solutions:

$$
\mathcal{D}_{j} \geq k-2 j+1 \text {. }
$$

To obtain an upper bound for $\mathcal{D}_{j}$, we shall use the fact that the sum of multiplicities of zeros of a theta-function of degree $2 k$ in any fundamental cell is $2 k$. Now, if we have $\mathcal{D}_{j}$ independent even theta-functions with common zeros of total multiplicity $4 j \leq 2 k$, by a linear combination of them we can obtain another theta-function whose zeros have multiplicity at least $4 j+2\left(\mathcal{D}_{j}-1\right)$. Since the latter number has to be $\leq 2 k$, it follows that $\mathcal{D}_{j} \leq k-2 j+1$ and consequently we obtain the expected result

$$
\mathcal{D}_{j}= \begin{cases}k-2 j+1 & \text { for } j \leq k / 2 \\ 0 & \text { otherwise }\end{cases}
$$

The case of odd spin $j$ can be treated in a similar way. Now we have $k-1$ independent odd theta-functions and $(4.12, b)$ gives $2 j-2$ independent conditions. Finally we obtain the same expression (4.15) for the $\mathcal{D}_{j}$.

\section{Knizhnik-Zamolodchikov-Bernard equations}

In Section 2 we have described how the states of Chern-Simons theory may be identified with holomorphic factors of (euclidean) Green functions for the WZW theory given by a formal functional integral. Using the functional integral representation, one may deduce equations that describe the behavior of the Green functions under changes of the conformal structure of the torus and of the insertion points. In the spherical topology, such relations are known as the Knizhnik-Zamolodchikov (KZ) equations [24]. They were generalized to the toroidal case in [4] and to general Riemann surfaces in [5]. They induce a connection which allows comparing spaces of Chern-Simons states for different punctured surfaces, see [33] [21] [16] [2]. We have included a brief heuristic derivation of the toroidal equations for the sake of completeness.

First, let us consider twisted toroidal Green functions of the WZW theory coupled to the $(0,1)$-component of the connection:

$$
\left\langle\Phi_{1}\left(z_{1}\right) \cdots \Phi_{N}\left(z_{N}\right)\right\rangle_{\eta, A^{01}}=\int \otimes_{n}\left(g_{1} g\right)\left(z_{n}\right)_{(n)} \exp \left[-k S_{W Z W}\left(g_{1} g, A\right)\right] D g .
$$

Field $g$ in (5.1) is periodic i.e. $g(z+2 \pi)=g(z+2 \pi \tau)=g(z)$ and $g_{1}(z+2 \pi)=g_{1}(z)$, $g_{1}(z+2 \pi \tau)=\eta g_{1}(z)$ with twist $\eta \in G^{\mathbb{C}}$. Also $A^{01}(z+2 \pi)=A^{01}(z), A^{01}(z+2 \pi \tau)=\operatorname{Ad}_{\eta} A^{01}(z)$. 
The WZW action for the twisted fields may be defined [12] so that if $g_{1}^{\prime}(q z)=\eta^{\prime} g_{1}^{\prime}(z), A^{01}=$ $g_{1} g_{1}^{\prime-1} \bar{\partial}\left(g_{1}^{\prime} g_{1}^{-1}\right)$ then

$$
S_{W Z W}\left(g_{1} g, A^{01}\right)=S_{W Z W}\left(g_{1}^{\prime} g\right)-S_{W Z W}\left(g_{1}^{\prime}\right)+S_{W Z W}\left(g_{1}, A^{01}\right) .
$$

In particular, for $g_{1}^{\prime}(z)=\mathrm{e}^{\sigma_{3}(z-\bar{z}) u /\left(2 \tau_{2}\right)} \equiv \eta_{u}^{-1} g_{1}^{\prime}(z+2 \pi \tau)$ and $g_{1}=1$, we have

$$
S_{W Z W}\left(g, A_{u}^{01}\right)=S_{W Z W}\left(g_{1}^{\prime} g\right)-\pi u^{2} / \tau_{2}
$$

and we obtain the following relation between the untwisted and twisted Green functions:

$$
\left\langle\Phi_{1}\left(z_{1}\right) \cdots \Phi_{N}\left(z_{N}\right)\right\rangle_{1, A^{01}}=e^{\pi k u^{2} / \tau_{2}} \prod_{n}\left(e^{\sigma_{3}\left(\bar{z}_{n}-z_{n}\right) u /\left(2 \tau_{2}\right)}\right)_{(n)}\left\langle\Phi_{1}\left(z_{1}\right) \cdots \Phi_{N}\left(z_{N}\right)\right\rangle_{\eta_{u}, 0} .
$$

As we saw before, Chern-Simons states $\Psi\left(A^{01}\right)$ have the same transformation properties under chiral gauge transformation as $\langle\cdots\rangle_{1, A^{01}}$. Comparing (5.3) and (4.1), one infers that, similarly, maps $\gamma(u)$ correspond to $\langle\cdots\rangle_{\eta_{u}, 0}$ for $\eta_{u}=\exp \left(2 \pi i u \sigma_{3}\right)$.

Eq. (5.2) implies the following Ward identity for the Green functions:

$$
\begin{aligned}
\left\langle\Phi_{1}\left(z_{1}\right) \cdots \Phi_{N}\left(z_{N}\right)\right\rangle_{\eta, A^{01}}= & \mathrm{e}^{k\left(S_{W Z W}\left(g_{1}^{\prime}\right)-S_{W Z W}\left(g_{1}, A^{01}\right)\right)} \\
& \cdot \prod_{n}\left(g_{1} g_{1}^{\prime-1}\right)\left(z_{n}\right)_{(n)}\left\langle\Phi_{1}\left(z_{1}\right) \cdots \Phi_{N}\left(z_{N}\right)\right\rangle_{\eta^{\prime}, 0} .
\end{aligned}
$$

On the other hand, correlations $\langle\cdots\rangle_{\eta, A}$ are generating functionals for Kac-Moody currents. For example,

$$
\left\langle J^{a}(z) J^{b}(w) \Phi_{1} \cdots \Phi_{N}\right\rangle_{\eta, 0}=\left.(2 \pi i)^{2} \frac{\delta^{2}}{\delta\left(A^{01}\right)^{a}(z) \delta\left(A^{01}\right)^{b}(w)}\left\langle\Phi_{1} \cdots \Phi_{N}\right\rangle_{\eta, A}\right|_{A=0} .
$$

where $A^{01}=\sum_{a}\left(A^{01}\right)^{a} t^{a}$. We shall normalize the Lie algebra generators $t^{a}$ so that $\operatorname{tr} t^{a} t^{b}=\frac{1}{2} \delta^{a b}$. Identity (5.4) allows to compute the Green functions with two current insertions if one solves

$$
\begin{aligned}
A^{01} & =h^{-1} \bar{\partial} h, \\
h(z+2 \pi) & =h(z), \\
h(z+2 \pi \tau) & =\eta^{\prime} h(z) \eta^{-1}
\end{aligned}
$$

for $h$ and $\eta^{\prime}$ to the second order in $A^{01}$. The latter may be expressed in terms of the fundamental solution $\omega(z, w \mid \eta)$ of the operator $\bar{\partial}$ with boundary condition

$$
\begin{gathered}
\omega(z+2 \pi, w \mid \eta)=\omega(z, w \mid \eta), \\
\omega(z+2 \pi \tau, w \mid \eta)=\operatorname{Ad}_{\eta} \omega(z, w \mid \eta)-i .
\end{gathered}
$$

More explicitly,

$$
\omega(z, w \mid \eta)=\frac{i \mathrm{e}^{i w}}{\mathrm{e}^{i z}-\mathrm{e}^{i w}}+\sum_{r=1}^{\infty}\left(\frac{i q^{r}}{A d_{\eta^{-1}}-q^{r}} \mathrm{e}^{i r(w-z)}-\frac{i q^{r}}{A d_{\eta}-q^{r}} \mathrm{e}^{i r(z-w)}\right) .
$$


where $q \equiv \mathrm{e}^{2 \pi i \tau}$. Now solutions of $(5.6, \mathrm{a}-\mathrm{c})$ are given by

$$
\begin{aligned}
\eta^{\prime} \eta^{-1}=\exp \left[\frac{1}{2 \pi} \int d w A^{01}(w)\right. & \\
& \left.+\frac{i}{8 \pi^{2}} \iint\left[\omega(z, w \mid \eta) d w A^{01}(w), d z A^{01}(z)\right]+\mathcal{O}\left(A^{3}\right)\right] \\
h(z)=\exp \left[\frac{i}{4 \pi} \int\left(\omega(z, w \mid \eta)+\omega\left(z, w \mid \eta^{\prime}\right)\right) d w A^{01}(w)\right. & \left.-\frac{1}{8 \pi^{2}} \iint \omega(z, y \mid \eta)\left[\omega(y, w \mid \eta) d w A^{01}(w), d y A^{01}(y)\right]+\mathcal{O}\left(A^{3}\right)\right] .
\end{aligned}
$$

Using those expressions on the right hand side of (5.4) one obtains for the Green functions with two current insertions:

$$
\begin{aligned}
\left\langle J^{a}(z) J^{b}(w)\right. & \left.\Phi_{1} \cdots \Phi_{N}\right\rangle_{\eta, 0}= \\
& \frac{1}{2}\left(\left[\sum_{n=1}^{N} \omega\left(z_{n}, z \mid \eta\right) t_{(n)}^{a}+i \mathcal{L}_{t^{a}}, \sum_{n=1}^{N} \omega\left(z_{n}, w \mid \eta\right) t_{(n)}^{b}+i \mathcal{L}_{t^{b}}\right]_{+}\right. \\
& -\sum_{n=1}^{N}\left(\omega\left(z_{n}, z \mid \eta\right)\left[\omega(z, w \mid \eta) t_{(n)}^{b}, t_{(n)}^{a}\right]+\omega\left(z_{n}, w \mid \eta\right)\left[\omega(w, z \mid \eta) t_{(n)}^{a}, t_{(n)}^{b}\right]\right) \\
& +i \mathcal{L}_{\left[t^{a}, \omega(z, w \mid \eta) t^{b}\right]}+i \mathcal{L}_{\left[t^{b}, \omega(w, z \mid \eta) t^{a}\right]} \\
& \left.-k \operatorname{tr}\left(t^{a} \partial_{z} \omega(z, w \mid \eta) t^{b}+t^{b} \partial_{w} \omega(w, z \mid \eta) t^{a}\right)\right)\left\langle\Phi_{1} \cdots \Phi_{N}\right\rangle_{\eta, 0}
\end{aligned}
$$

where $\omega$ in action on $t_{(n)}^{a}$ should be taken in the same representation, [, $]_{+}$stands for the anticommutator and $\mathcal{L}_{X}$ for the Lie derivative in the direction of the element $X$ of the Lie algebra of $G, \mathcal{L}_{X} f(\eta)=d /\left.d t\right|_{t=0} f\left(\mathrm{e}^{t X} \eta\right)$.

The energy-momentum tensor of the WZW theory is given by the Sugawara construction 28:

$$
\left(k+h^{\vee}\right) T(w)=\lim _{x \rightarrow w}\left(\sum_{a} J^{a}(z) J^{a}(w)-\frac{k \operatorname{dim} G}{2(z-w)^{2}}\right) .
$$

Using (5.9) specialized to the $S U(2)$ case and to $\eta_{u}=\exp \left(2 \pi i u \sigma_{3}\right)$, we obtain after a straightforward algebra explained in Appendix B $\left(t^{a} \equiv \frac{1}{2} \sigma_{a}, t^{ \pm} \equiv t^{1} \pm i t^{2}\right)$ :

$$
\begin{aligned}
& \left\langle T(w) \Phi_{1} \cdots \Phi_{N}\right\rangle_{\eta_{u}, 0}=\frac{1}{k+2}\left[\left(\sum_{n=1}^{N} \omega\left(z_{n}, w \mid \eta_{u}\right) t_{(n)}^{3}+\frac{1}{4 \pi} \partial_{u}\right)^{2}\right. \\
& +\frac{1}{2}\left[\sum_{n=1}^{N}\left(\omega\left(z_{n}, w \mid \eta_{u}\right)+\frac{i}{1-\mathrm{e}^{4 \pi i u}}\right) t_{(n)}^{+}, \sum_{n=1}^{N}\left(\omega\left(z_{n}, w \mid \eta_{u}\right)+\frac{i}{1-\mathrm{e}^{-4 \pi i u}}\right) t_{(n)}^{-}\right]_{+} \\
& +i\left(1+2 \sum_{r=0}^{\infty} \frac{q^{r}}{\mathrm{e}^{4 \pi i u}-q^{r}}-2 \sum_{r=1}^{\infty} \frac{q^{r}}{\mathrm{e}^{-4 \pi i u}-q^{r}}\right)\left(\sum_{n=1}^{N} \omega\left(z_{n}, w \mid \eta_{u}\right) t_{(n)}^{3}+\frac{1}{4 \pi} \partial_{u}\right)
\end{aligned}
$$




$$
\left.-k \sum_{r=1}^{\infty}\left(\frac{r q^{r}}{1-q^{r}}+\frac{r q^{r}}{\mathrm{e}^{4 \pi i u}-q^{r}}+\frac{r q^{r}}{\mathrm{e}^{-4 \pi i u}-q^{r}}\right)+\frac{3 k}{24}\right]\left\langle\Phi_{1} \cdots \Phi_{N}\right\rangle_{\eta_{u}, 0} \cdot
$$

On the other hand, the Green functions involving the insertion of the energy-momentum tensor and of the primary fields follow from the covariance of the theory under diffeomorphisms $z \mapsto z+\zeta(z)$ such that $\zeta(z+2 \pi)=\zeta(z)$ and $\zeta(z+2 \pi \tau)=\zeta(z)+2 \pi\left(\tau^{\prime}-\tau\right)$. To the linear order in $\zeta$,

$$
\begin{aligned}
& \left.\left\langle\Phi_{1}\left(z_{1}^{\prime}\right) \cdots \Phi_{N}\left(z_{N}^{\prime}\right)\right\rangle_{\eta, 0}\right|_{\tau}+\left.\frac{1}{2 \pi i} \int g_{\bar{w} \bar{w}}(w)\left\langle T(w) \Phi_{1}\left(z_{1}\right) \cdots \Phi_{N}\left(z_{N}\right)\right\rangle_{\eta, 0}\right|_{\tau} d w d \bar{w} \\
= & \left.\left(1+\partial_{z} \zeta\left(z_{1}\right)\right)^{\Delta_{1}} \ldots\left(1+\partial_{z} \zeta\left(z_{N}\right)\right)^{\Delta_{N}}\left\langle\Phi_{1}\left(z_{1}+\zeta\left(z_{1}\right)\right) \cdots \Phi_{N}\left(z_{N}+\zeta\left(z_{N}\right)\right)\right\rangle_{\eta, 0}\right|_{\tau^{\prime}}
\end{aligned}
$$

where the second term on the left arises because the metric $d z^{\prime} d \bar{z}^{\prime}$ develops a non-trivial $g_{\bar{z} \bar{z}}$ part in new variables:

$$
g_{\bar{z} \bar{z}}=\partial_{\bar{z}} \zeta
$$

$\Delta_{n}$ are the conformal weights of fields $\Phi_{n}$. Solving eq. (5.13) for $\zeta$ to the first order in $g_{\bar{z} \bar{z}}$, we obtain

$$
\begin{gathered}
\zeta(z)=\frac{i}{2 \pi} \int \omega(z, w) g_{\bar{w} \bar{w}}(w) d w d \bar{w} \\
\tau^{\prime}=\tau+\frac{1}{4 \pi^{2}} \int g_{\bar{z} \bar{z}}(z) d z d \bar{z}
\end{gathered}
$$

where $\omega(z, w)$ is as in (5.7) but with $\mathrm{Ad}_{\eta}$ replaced by 1. Inserting eqs. (5.14), (5.15) into (5.12), we obtain

$$
\begin{aligned}
\langle T(w) & \left.\Phi_{1} \cdots \Phi_{N}\right\rangle_{\eta_{u}, 0} \\
= & \left(\sum_{n=1}^{N}\left(\Delta_{n} \partial_{w} \omega\left(z_{n}, w\right)-\omega\left(z_{n}, w\right) \partial_{z_{n}}\right)-\frac{1}{2 \pi i} \partial_{\tau}\right)\left\langle\Phi_{1} \cdots \Phi_{N}\right\rangle_{\eta_{u}, 0}
\end{aligned}
$$

The comparison of the right hand sides of (5.11) and (5.16), gives an identity satisfied by the Green functions. It may be rewritten as an identity for the holomorphic factors $\gamma$ we have introduced earlier:

$$
\nabla(w) \gamma=0
$$

where

$$
\begin{aligned}
\nabla(w) & =(k+2)\left(\sum_{n=1}^{N}\left(-\Delta_{n} \partial_{w} \omega\left(z_{n}, w\right)+\omega\left(z_{n}, w\right) \partial_{z_{n}}\right)\right) \\
& +\Pi(u, \tau)^{-1}\left\{\frac{k+2}{2 \pi i} \partial_{\tau}+\left(\sum_{n=1}^{N} \omega\left(z_{n}, w\right) t_{(n)}^{3}+\frac{1}{4 \pi} \partial_{u}\right)^{2}\right\} \Pi(u, \tau)
\end{aligned}
$$




$$
+\frac{1}{2}\left[\sum_{n=1}^{N}\left(\omega\left(z_{n}, w \mid \eta_{u}\right)+\frac{i}{1-\mathrm{e}^{4 \pi i u}}\right) t_{(n)}^{+}, \sum_{n=1}^{N}\left(\omega\left(z_{n}, w \mid \eta_{u}\right)+\frac{i}{1-\mathrm{e}^{-4 \pi i u}}\right) t_{(n)}^{-}\right]_{+}
$$

and

$$
\Pi(u, \tau)=q^{1 / 8} \sin (2 \pi u) \prod_{r}\left(1-q^{r}\right)\left(1-e^{4 \pi i u} q^{r}\right)\left(1-e^{-4 \pi i u} q^{r}\right)
$$

corresponds to the solution of (5.17) for the case of $k=2$ and only one insertion of spin $j=1$.

Let us discuss the structure of eq. (5.17). Since $\gamma$ is $w$-independent, the left hand side is, a priori, a meromorphic function of $w$ with poles of at most second order at the insertion points $z_{n}$. Equating the coefficients at $\left(w-z_{n}\right)^{-2}$ to zero, we infer that $\Delta_{n}=j_{n}\left(j_{n}+1\right) /(k+2)$. Similarly, from the vanishing of the residues, we obtain equations for the derivatives $\partial_{z_{n}} \gamma$. They generalize the KZ equations to the toroidal case. Finally, the regular part of the left-hand side of eq. (5.17) is holomorphic and periodic in $w$ so constant and it gives the derivative $\partial_{\tau} \gamma$.

Explicitly, the different components of the connection are:

$$
\begin{aligned}
\nabla_{z_{n}}= & \partial_{z_{n}}+\frac{1}{2 \pi(k+2)} \Pi(u, \tau)^{-1} \partial_{u} \Pi(u, \tau) t_{(n)}^{3} \\
& +\frac{1}{k+2} \sum_{m \neq n}\left(t_{(n)}^{3}\left(2 \omega\left(z_{m}, z_{n}\right)+1\right) t_{(m)}^{3}+t_{(n)}^{-}\left(\omega\left(z_{m}, z_{n} \mid \eta_{u}\right)+\frac{i}{1-\mathrm{e}^{4 \pi i u}}\right) t_{(m)}^{+}\right. \\
& \left.\quad+t_{(n)}^{+}\left(\omega\left(z_{m}, z_{n} \mid \eta_{u}\right)+\frac{i}{1-\mathrm{e}^{-4 \pi i u}}\right) t_{(m)}^{-}\right)
\end{aligned}
$$

and

$$
\begin{aligned}
\nabla_{\tau} & =\Pi(u, \tau)^{-1}\left(\partial_{\tau}+\frac{i}{8 \pi(k+2)} \partial_{u}^{2}\right) \Pi(u, \tau) \\
& +\frac{2 \pi}{k+2} \sum_{m, n=1}^{N}\left(t_{(n)}^{3} \widetilde{\omega}\left(z_{m}, z_{n} \mid \eta_{u}\right) t_{(m)}^{3}+\frac{1}{2}\left[t_{(n)}^{-},\left(\widetilde{\omega}\left(z_{m}, z_{n} \mid \eta_{u}\right)+\frac{i \mathrm{e}^{4 \pi i u}}{\left(1-\mathrm{e}^{4 \pi i u}\right)^{2}}\right) t_{(m)}^{+}\right]_{+}\right)
\end{aligned}
$$

where

$$
\widetilde{\omega}(z, w \mid \eta)=\sum_{r=1}^{\infty}\left(\frac{i q^{r} A d_{\eta^{-1}}}{\left(A d_{\eta^{-1}}-q^{r}\right)^{2}} \mathrm{e}^{i r(w-z)}+\frac{i q^{r} A d_{\eta}}{\left(A d_{\eta}-q^{r}\right)^{2}} \mathrm{e}^{i r(z-w)}\right) .
$$

To arrive at expressions (5.20) and (5.21) we have used some algebraic relations satisfied by $\omega$ that are listed in Appendix C.

Eq. (5.17) has been obtained by manipulating formal functional integrals. Now, we would like to prove that $\nabla(w)$ defines a flat holomorphic connection in the bundle of toroidal ChernSimons states. In analogy to the genus zero case where the KZ equations give holomorphic connection in the trivial bundle of invariant tensors [23], one could expect that $\nabla(w)$ defines a holomorphic connection already in the vector bundle $W_{\underline{j}}$ with the base-space $\mathcal{T}_{N}$ of points $(\underline{z}, \tau)$ and the fibers composed of maps $\gamma$ satisfying conditions (4.2). This is, however, not the case: although formally the connection given by $\nabla(w)$ is flat, it does not preserve the analyticity (in $u$ ) of maps $\gamma$ producing, in general, first order poles at $u \in(\mathbb{Z}+\tau \mathbb{Z}) / 2$ when acting on $\gamma$. This 
is not a problem if $\gamma$ takes values in $W_{\underline{j}}^{f r}$ (i.e. if $\gamma(\underline{z}, \tau)$ satisfy additionally conditions (4.10)). We shall see that in this case $\nabla(w) \gamma$ is analytic as a function of $u$ and, for fixed $(\underline{z}, \tau)$ and $w \neq z_{n}$, belongs again to $W_{\underline{j}}^{f r}$. Consequently, $\nabla(w)$ will define a flat holomorphic connection on $W_{\underline{j}}^{f r}$ provided that we prove that the latter is a holomorphic subbundle of $W_{\underline{j}}$. This is precisely why we need to define the connection first on a bigger bundle. Given such a connection with parallel transport preserving conditions (4.10), it would follow that the spaces of solutions of (4.10) have dimension independent of $(\underline{z}, \tau)$ which is all we need in order to show that $W_{j}^{f r}$ is a subbundle of $W_{j}$. It is possible to obtain from $\nabla(w)$ a well defined connection enlarging bundle $W_{\underline{j}}$ by admitting $\gamma$ 's with poles at $u=(\mathbb{Z}+\tau \mathbb{Z}) / 2$. This gives, however, a connection in the bundle with an infinite-dimensional fiber where the existence of the parallel transport does not come for free (it becomes a hard PDE problem rather than an easy ODE one). Instead, we shall stay in the finite-dimensional setup but modify $\nabla(w)$ to $\widetilde{\nabla}(w)$ by subtracting the pole term so that the modifications disappear in the action on $W_{\underline{j}}^{f r}$-valued $\gamma$ 's and that $\widetilde{\nabla}(w)$ gives a well defined (holomorphic) connection on $W_{\underline{j}}$.

First, let us show that if $\gamma$ takes values $W_{j}^{f r}$ then $\nabla(w) \gamma$, as a function of $u$, has no poles at $u=0$. This may be checked directly but may be also understood by the following argument. If $\gamma$ satisfies conditions (4.10) then it defines a global state $\Psi$. In particular, for $M \in \operatorname{sl}(2, \mathbb{C})$ and $A_{M}^{01}$ as in (3.10), we may write

$$
\Psi\left(A_{M}^{01}\right)=\mathrm{e}^{\frac{1}{2} \pi k \operatorname{tr}\left(M^{2}\right)} \prod_{n}\left(\mathrm{e}^{\sigma_{3}\left(\bar{z}_{n}-z_{n}\right) /\left(2 \tau_{2}\right)}\right)_{(n)} \gamma(M)
$$

so that, with a slight abuse of notation, $\gamma(u)$ coincides with $\gamma(M)$ for $M=u \sigma_{3}$, see (4.1). Due to (4.10), $\gamma(M)$ is analytic in $M$. Besides, it depends only on $\mathrm{e}^{2 \pi i M} \equiv \eta$. Indeed, if $\mathrm{e}^{2 \pi i M_{1}}=\mathrm{e}^{2 \pi i M_{2}}$ then $A_{M_{1}}^{01}={ }^{h} A_{M_{2}}^{01}$ for $h(z)=\exp \left[\frac{1}{2} \tau_{2}^{-1}(\bar{z}-z) M_{1}\right] \exp \left[\frac{1}{2} \tau_{2}^{-1}(z-\bar{z}) M_{2}\right]$. Application of the basic covariance property (2.5) of the states gives the equality $\gamma\left(M_{1}\right)=\gamma\left(M_{2}\right)$. Since the exponential map $M \mapsto \eta$ may be locally inverted around $M=0$, it follows that $\gamma(\eta)$ is analytic around $\eta=1$ (in fact it is even globally analytic on $S L(2, \mathbb{C})$ ). Now the terms in $\nabla(w)$ with the poles at $u=0$ came from rewriting $\mathcal{L}_{t^{ \pm}}$and $\mathcal{L}_{t^{ \pm}} \mathcal{L}_{t^{\mp}}$ in (5.9), see Appendix C. In $\nabla(w) \gamma(u)$, they may be reabsorbed into $\left.\mathcal{L}_{t^{ \pm}} \gamma(\eta)\right|_{\eta=\eta_{u}}, \mathcal{L}_{t^{ \pm}} \omega\left(z_{n}, w \mid \eta_{u}\right)$ and $\left.\mathcal{L}_{t^{ \pm}} \mathcal{L}_{t \mp} \gamma(\eta)\right|_{\eta=\eta_{u}}$, all three regular in $u$ around $u=0$.

To show that the other poles in $u$ are absent in $\nabla(w) \gamma$ when $\gamma$ satisfies conditions (4.10), we shall use operators $\mathcal{Q}_{i}, i=1,2$, essentially extending the action (2.5) of gauge transformations to the case of "almost" gauge transformations $h_{v}$ for $v=\frac{1}{2}$ and $v=\frac{\tau}{2}$, see (3.8) (for the later convenience we shall use the subindex $\underline{j}$, in the notation for $\mathcal{Q}_{i}$ 's, to keep track of the insertions).

$$
\begin{aligned}
& \mathcal{Q}_{\underline{j}, 1} \gamma(u)=\gamma(u+1 / 2), \\
& \mathcal{Q}_{\underline{j}, 2} \gamma(u)=\mathrm{e}^{2 \pi i k(u+\tau / 4)} \prod_{n} \mathrm{e}^{-i z_{n} t_{(n)}^{3}} \gamma(u+\tau / 2)
\end{aligned}
$$

and they act at the space of analytic maps $\mathbb{C} \backslash(\mathbb{Z}+\tau \mathbb{Z}) / 2 \ni u \mapsto \gamma(u) \in \otimes V_{j_{n}}$. We shall also need

$$
\mathcal{P}_{\underline{j}, i} \quad=\mathcal{Q}_{\underline{j}, i}^{2} \quad \text { for } i=1,2,
$$




$$
\begin{aligned}
& \mathcal{P}_{\underline{j}, 3}=\prod_{n} \mathrm{e}^{i t_{(n)}^{3}}, \\
& \mathcal{P}_{\underline{j}, 4} \gamma(u)=\prod_{n} \Omega_{(n)} \gamma(-u),
\end{aligned}
$$

with $\Omega$ the generator of the Weyl group of $S L(2, \mathbb{C})$.

These operators satisfy the algebra

$$
\begin{aligned}
& {\left[\mathcal{Q}_{j, 1}, \mathcal{Q}_{\underline{j}, 2}\right]=0,} \\
& {\left[\mathcal{Q}_{j, i}, \mathcal{P}_{\underline{j}, 3}\right]=0,} \\
& \mathcal{Q}_{\underline{j}, i} \mathcal{P}_{\underline{j}, 4}=\mathcal{P}_{\underline{j}, 4} \mathcal{Q}_{\underline{j}, i}^{-1}, \\
& \mathcal{P}_{\underline{j}, 3} \mathcal{P}_{\underline{j}, 4}=\mathcal{P}_{\underline{j}, 4} \mathcal{P}_{\underline{j}, 3}^{-1}
\end{aligned}
$$

and conditions (4.2) can be written as

$$
\left(\mathcal{P}_{\underline{j}, i}-1\right) \gamma=0 \quad \text { for } i=1, \ldots, 4
$$

Defining

$$
D_{\underline{j}, \alpha}^{L}=\widehat{D}_{\underline{j}, 0}^{L} \mathcal{Q}_{\underline{j}, 1}^{a} \mathcal{Q}_{\underline{j}, 2}^{b} \quad \text { for } \quad \alpha \equiv(a+b \tau) / 2=0,1 / 2, \tau / 2,(\tau+1) / 2,
$$

we may rewrite the conditions of (4.10) in an equivalent way using operators $D_{\underline{j}, \alpha}^{L}$ instead of $\widehat{D}_{\underline{j}, \alpha}^{L}$. From (5.25), (5.26) and (5.27) it is clear that $\mathcal{Q}_{\underline{j}, i}$ preserve spaces $W_{\underline{j}}(\underline{z}, \tau)$ and $W_{\underline{j}}^{f r}(\underline{z}, \tau)$. Also

$$
\begin{array}{ll}
{\left[\mathcal{Q}_{j}, i, \nabla(w)\right]=0} & \text { for } i=1,2, \\
{\left[\mathcal{P}_{\underline{j}, i}^{-}, \nabla(w)\right]=0} & \text { for } i=1, \cdots, 4
\end{array}
$$

so that

$$
\begin{aligned}
\nabla(w) \gamma(u+(p+r \tau) / 2) & =\mathrm{e}^{-2 \pi i k\left(r u+r^{2} \tau / 4\right)} \otimes_{n} \mathrm{e}^{i n z_{n} t_{(n)}^{3}} \mathcal{Q}_{\underline{j}, 1}^{p} \mathcal{Q}_{\underline{j}, 2}^{r} \nabla(w) \gamma(u) \\
& =\mathrm{e}^{-2 \pi i k\left(r u+r^{2} \tau / 4\right)} \otimes_{n} \mathrm{e}^{i n z_{n} t_{(n)}^{3} \nabla(w) \mathcal{Q}_{\underline{j}, 1}^{p} \mathcal{Q}_{\underline{j}, 2}^{r} \gamma(u)}
\end{aligned}
$$

As $\nabla(w) \gamma$ is analytic around $u=0$ provided $\gamma$ is a Chern-Simons state, it follows that, in this case, $\nabla(w) \gamma$ is entire as a function of $u$.

Let us pass to redefining $\nabla(w)$ by subtracting "by hand" its poles in such a way that the modifications vanish when acting on $W_{\underline{j}}^{f r}$-valued $\gamma^{\prime}$ s and the new regular $\widetilde{\nabla}(w)$ defines a holomorphic connection in $W_{\underline{j}}$. This may be done by means of operators $R_{n}, R_{0}$,

$$
\begin{aligned}
& R_{n} \gamma(u)=\frac{i}{4} \sum_{r \in \mathbb{Z}} \mathrm{e}^{2 \pi i k\left(r^{2} \tau-2 r u\right)}\left(\frac{q^{r}}{\mathrm{e}^{2 \pi i u}-q^{r}}-\frac{q^{-r}}{\mathrm{e}^{-2 \pi i u}-q^{-r}}\right) \\
&\left(\prod_{m} \mathrm{e}^{\left.2 i r z_{m} t_{(m)}^{3}\right)}\right)\left(t_{(n)}^{+} \sum_{m} t_{(m)}^{-}-t_{(n)}^{-} \sum_{m} t_{(m)}^{+}\right) \gamma(0) \quad \text { for } \quad n=1, \ldots, N, \\
& R_{0} \gamma(u)=\frac{1}{8} \sum_{r \in \mathbb{Z}} \frac{\mathrm{e}^{2 \pi i k\left(r^{2} \tau-2 r u\right)}}{\left(\mathrm{e}^{2 \pi i u}-q^{r}\right)\left(\mathrm{e}^{-2 \pi i u}-q^{-r}\right)}\left(\prod_{m} \mathrm{e}^{2 i r z_{m} t_{(m)}^{3}}\right)\left[\sum_{m} t_{(m)}^{+}, \sum_{m} t_{(m)}^{-}\right]_{+} \gamma(0)
\end{aligned}
$$




$$
\begin{array}{r}
+\frac{i}{32 \pi} \sum_{r \in \mathbb{Z}} \mathrm{e}^{2 \pi i k\left(r^{2} \tau-2 r u\right)}\left(\frac{q^{r}}{\mathrm{e}^{2 \pi i u}-q^{r}}-\frac{q^{-r}}{\mathrm{e}^{-2 \pi i u}-q^{-r}}\right) \\
\left(\prod_{m} \mathrm{e}^{\left.2 i r z_{m} t_{(m)}^{3}\right)}\right)\left(\left[\sum_{m} t_{(m)}^{+}, \sum_{m} t_{(m)}^{-}\right]_{+}-4\right) \partial_{u} \gamma(0)
\end{array}
$$

which vanish on $W_{\underline{j}}^{f r}(\underline{z}, \tau)$ (see Appendix D) and make

$$
\widetilde{\nabla}(w)=\nabla(w)-\sum_{a, b=0,1} \mathcal{Q}_{\underline{j}, 1}^{a} \mathcal{Q}_{\underline{j}, 2}^{b}\left(\frac{1}{2 \pi i} R_{0}-\sum_{n=1}^{N} \omega\left(z_{n}, w\right) R_{n}\right) \mathcal{Q}_{\underline{j}, 1}^{-a} \mathcal{Q}_{\underline{j}, 2}^{-b}
$$

regular when acting on $W_{\underline{j}}$. Note that $\left(\mathcal{P}_{i}-1\right) R_{n}=0$ for $i=1, \ldots, 4$ and $n=0, \ldots, N$ so that $\widetilde{\nabla}(w)$ actually defines a connection on $W_{j}$. This connection is not flat any more but, as may be checked, its curvature vanishes on $W_{\underline{j}}^{f r}$-valued sections.

Now we want to show that the connection on $W_{\underline{j}}$ defines by parallel transport an isomorphism between spaces $W_{\underline{j}}^{f r}(\underline{z}, \tau)$ for different $(\underline{z}, \tau)$. This comes from the following relations obtained by commuting $\bar{D}_{j, \alpha}^{L}$ and $\widetilde{\nabla}(w)$ or its components $\widetilde{\nabla}_{z_{n}}, \widetilde{\nabla}_{\tau}$ (a long but straightforward calculation which is detailèd in Appendix D)

$$
\begin{aligned}
& D_{\underline{j}, \alpha}^{L} \widetilde{\nabla}_{z_{n}} \gamma=\partial_{z_{n}} D_{\underline{j}, \alpha}^{L} \gamma+\sum_{\beta, L^{\prime}} A_{n, \alpha L^{\prime}}^{\beta L} D_{\underline{j}, \beta}^{L^{\prime}} \gamma \quad \text { for } n=1, \ldots, N, \\
& D_{\underline{j}, \alpha}^{L} \widetilde{\nabla}_{\tau} \gamma=\partial_{\tau} D_{\underline{j}, \alpha}^{L} \gamma+\sum_{\beta, L^{\prime}} A_{0, \alpha L^{\prime}}^{\beta L} D_{\underline{j}, \beta}^{L^{\prime}} \gamma,
\end{aligned}
$$

where for $|L|<J$ and $n=0, \ldots, N, A_{n, \alpha L^{\prime}}^{\beta L}$ is different from zero only if $\left|L^{\prime}\right|<J$. Thus if $D_{\underline{j}, \alpha}^{L} \gamma$ vanish for $|L|<J$ at the initial point of the $\widetilde{\nabla}$-horizontal curve in $W_{\underline{j}}$ they also do along the whole curve. As a consequence, dimensions of spaces $W_{\underline{j}}^{f r}(\underline{z}, \tau)$ are independent of points $(\underline{z}, \tau)$ and $W_{\underline{j}}^{f r}$ is a holomorphic subbundle of $W_{\underline{j}}$ with a holomorphic (flat) connection $\nabla$ coinciding with the restriction of $\widetilde{\nabla}$.

Using the properties of $\omega(z, w \mid \eta)$ under modular transformations, listed in Appendix $\mathrm{C}$, it is easy to check that the action of the modular group $\Gamma_{N}$ almost preserves the connection $\widetilde{\nabla}$ : $\widetilde{\nabla}_{z_{n}}$ is preserved but the pullback of $\widetilde{\nabla}_{\tau}$ is shifted by $c(c \tau+d)^{-1} \sum_{n} \Delta_{n}$ (not very precisely, we may say that $\gamma$ 's transform as the product of $\Delta_{n}$-forms). As the result, $\widetilde{\nabla}$ and $\nabla$ define canonically only projective connections on $W_{\underline{j}} / \Gamma_{N}$ and $W_{\underline{j}}^{f r} / \Gamma_{N}$, respectively.

\section{Factorization}

In this Section we would like to derive relations between the spaces of blocks with different number of insertions. In order to do so we shall study, as in [17], the behavior of our bundle when two punctures come together. Taking the punctures at $\left(z_{1}=z_{2}+\zeta^{2(k+2)}, z_{2}, \cdots, z_{N}\right)$, we 
shall let $\zeta \rightarrow 0$. We shall study the case when $j_{1}=1 / 2$ and the rest are positive integrable spins i.e. $0<j_{n} \leq k / 2 n=2, \cdots, N$.

Let us consider the connection

$$
\nabla_{\zeta}=\frac{\zeta^{2 k+3}}{\pi i} \oint_{C_{1}} \frac{d w}{w} \widetilde{\nabla}(w)
$$

where the path $C_{1}$ encloses clockwise only insertion at $z_{1}$.

$$
\nabla_{\zeta}=\partial_{\zeta}-2 \zeta^{-1}\left(2 t_{(1)}^{3} t_{(2)}^{3}+t_{(1)}^{+} t_{(2)}^{-}+t_{(1)}^{-} t_{(2)}^{+}\right)+\mathcal{O}\left(\zeta^{2 k+3}\right)
$$

and the connection has a singularity when $\zeta \rightarrow 0$. To regularize it we must diagonalize the singular term. The decomposition

$$
\bigotimes_{n} V_{j_{n}} \cong \bigoplus_{j=j_{2} \pm 1 / 2} \operatorname{Inv}\left(V_{1 / 2} \otimes V_{j_{2}} \otimes V_{j}\right) \otimes\left(V_{j} \otimes V_{j_{3}} \cdots \otimes V_{j_{N}}\right)
$$

does the job. Explicitly,

$$
\gamma(u)\left(\left(v_{n}\right)\right)=\left.\left.\sum_{j=j_{2} \pm 1 / 2} \sum_{l=0}^{2 j} \partial_{v}^{2 j-l}\right|_{v=0} P_{C G}^{1 / 2 j_{2} j}\left(v_{1}, v_{2}, v\right) \partial_{v^{\prime}}^{l}\right|_{v^{\prime}=0} \widehat{\gamma}_{j j_{3} \cdots j_{N}}(u)\left(v^{\prime}, v_{3}, \cdots, v_{N}\right)
$$

where the Clebsch-Gordan invariant tensors are given by

$$
P_{C G}^{j_{1} j_{2} j_{3}}\left(v_{1}, v_{2}, v_{3}\right)=\left(v_{1}-v_{2}\right)^{j_{12}}\left(v_{1}-v_{3}\right)^{j_{13}}\left(v_{2}-v_{3}\right)^{j_{23}}
$$

if $j_{12}=j_{1}+j_{2}-j_{3}, j_{13}=j_{1}+j_{3}-j_{2}$ and $j_{23}=j_{2}+j_{3}-j_{1}$ are non-negative integers and by zero otherwise.

Now we perform a gauge transformation in the $\widehat{\gamma}$ 's by the isomorphism $($ at $\zeta \neq 0)$

$$
\begin{aligned}
\varphi_{\zeta} \gamma & =\left(\widetilde{\gamma}_{\underline{j}^{+}}, \widetilde{\gamma}_{\underline{j}^{-}}\right) \\
& =\left(\zeta^{-2 j_{2}} \widehat{\gamma}_{\underline{j}^{+}}, \zeta^{2 j_{2}+2} \widehat{\gamma}_{\underline{j}^{-}}\right) .
\end{aligned}
$$

where $\underline{j}^{ \pm}$stands for insertions of spins $j_{2} \pm 1 / 2, j_{3}, \ldots, j_{N}$. In this way the connection $\widetilde{\nabla}_{\zeta}=$ $\varphi_{\zeta}^{-1} \nabla_{\zeta} \varphi_{\zeta}$ becomes regular at $\zeta=0$.

On the space of states we have

$$
\varphi_{\zeta} W_{\underline{j}}^{f r}\left(\left(z_{2}+\zeta^{2(k+2)}, z_{2}, \cdots, z_{N}\right), \tau\right)=\widetilde{W}_{\underline{j}}^{f r}(\zeta) .
$$

By the parallel transport with $\widetilde{\nabla}_{\zeta}$ to $\zeta=0$, we obtain space $\widetilde{W}_{j}^{f r}(0)$. Our next goal will be to show that this space coincides with $W_{\underline{j}^{+}}^{f r}\left(\left(z_{2}, \cdots, z_{N}\right), \tau\right) \oplus W_{\underline{j}^{-}}^{f r}\left(\left(z_{2}, \cdots, z_{N}\right), \tau\right)$. Let $\left(\widetilde{\gamma}_{\underline{j}^{+}}, \widetilde{\gamma}_{\underline{j}^{-}}\right)$, defined for small $|\zeta|$, be $\widetilde{\nabla}$-horizontal and with values in $\widetilde{W}_{\underline{j}}^{f r}(\zeta)$.

First $\left(\mathcal{P}_{j^{ \pm}, i}-1\right) \widetilde{\gamma}_{\underline{j}^{ \pm}}(\zeta)=0$ for $i=1,3,4$ and all $\zeta \neq 0$ so, by continuity, also for $\zeta=0$. As for $\mathcal{P}_{\underline{j}, 2}$ we have:

$$
\mathcal{P}_{\underline{j}, 2}=\mathrm{e}^{2 \pi i k(2 u+\tau)} \mathrm{e}^{\tau \partial_{u}} \exp \left[-2 i\left(z_{2}+\zeta^{2(k+2)}\right) t_{(1)}^{3}-2 i z_{2} t_{(2)}^{3}-\cdots-2 i z_{N} t_{(N)}^{3}\right]
$$




$$
=\widetilde{\mathcal{P}}_{\underline{j}, 2}+\mathcal{O}\left(\zeta^{2(k+2)}\right)
$$

where the $\mathcal{O}$-term is a linear operator and

$$
\widetilde{\mathcal{P}}_{\underline{j}, 2}=\mathrm{e}^{2 \pi i k(2 u+\tau)} \mathrm{e}^{\tau \partial_{u}} \exp \left[-2 i z_{2}\left(t_{(1)}^{3}+t_{(2)}^{3}\right)-2 i z_{3} t_{(3)}^{3}-\cdots-2 i z_{N} t_{(N)}^{3}\right] .
$$

Then

$$
\begin{aligned}
& \varphi_{\zeta}\left(\widetilde{\mathcal{P}}_{\underline{j}, 2}-1\right) \gamma\left(\left(z_{2}+\zeta^{2(k+2)}, z_{2}, \cdots, z_{N}\right), \tau\right) \\
= & \left(\left(\mathcal{P}_{\underline{j}^{+}, 2}-1\right) \widetilde{\gamma}_{\underline{j}^{+}}(\zeta),\left(\mathcal{P}_{\underline{j}^{-}, 2}-1\right) \widetilde{\gamma}_{\underline{j}^{-}}(\zeta)\right)=\mathcal{O}\left(\zeta^{2\left(k+1-j_{2}\right)}\right)
\end{aligned}
$$

and $\left(\mathcal{P}_{\underline{j}^{ \pm}, 2}-1\right) \widetilde{\gamma}_{\underline{j}^{ \pm}}(0)=0$.

We shall study the rest of the conditions that define the space of states i.e. those involving operators $D_{j, \alpha}^{L}$. Due to the homogeneity of maps $\gamma$, we may always take $L$ in $D_{j, \alpha}^{L}$ with one of the $l_{n}=\overline{0}$ and obtain an equivalent set of conditions. In the following we will take $L$ with $l_{2}=0$ and to remember this restriction we shall label such $L$ as $\widetilde{L}$.

Now we are going to examine the behavior of $D_{\underline{j}, \alpha}^{\widetilde{L}} \gamma$ near $\zeta=0$. Let $L^{n}\left(L_{n}\right)$ denote multiindex $L$ with $l_{n}$ increased (lowered) by 1 (if $l_{n}=0$ then terms with $L_{n}$ should be omitted).

$$
\begin{gathered}
D_{\underline{j}, \alpha}^{\widetilde{L}} \nabla_{\zeta} \gamma=\partial_{\zeta} D_{\underline{j}, \alpha}^{\widetilde{L}} \gamma-2 \zeta^{-1}\left[\left(j_{2}\left(1-2 l_{1}\right)-l_{1}\left(2-l_{1}\right)\right) D_{\underline{j}, \alpha}^{\widetilde{L}} \gamma\right. \\
\left.-l_{1}\left(2-l_{1}\right)\left(\left(j_{2}+1 / 2\right) D_{\underline{j}, \alpha}^{\widetilde{L}_{1}} \gamma-\sum_{n=3}^{N}\left(D_{\underline{j}, \alpha}^{\widetilde{L}_{1}^{n}} \gamma+\left(j_{n}-l_{n}\right) D_{\underline{j}, \alpha}^{\widetilde{L}_{1}} \gamma\right)\right)\right] \\
+\mathcal{O}\left(\zeta^{2 k+3}\right)
\end{gathered}
$$

which develops a singularity when $\zeta \rightarrow 0$. We have used the homogeneity of degree $J$ in variables $\left(v_{n}\right)$ of polynomial $\gamma$ and so:

$$
\sum_{n}\left(D_{\underline{j}, \alpha}^{L^{n}}+\left(l_{n}-j_{n}\right) D_{\underline{j}, \alpha}^{L}\right) \gamma=0
$$

The transformation

$$
\begin{array}{rlr}
\widetilde{D}_{\underline{j}, \alpha}^{\widetilde{L}} \gamma=\zeta^{-2 j_{2}} D_{\underline{j}, \alpha}^{\widetilde{L}} \gamma & \text { for } l_{1}=0, \\
\widetilde{D}_{\underline{j}, \alpha}^{L} \gamma=\zeta^{2\left(j_{2}+1\right)}\left(D_{\underline{j}, \alpha}^{\widetilde{L}} \gamma+\frac{1}{2} D_{\underline{j}, \alpha}^{\widetilde{L}_{1}} \gamma\right. & \\
\left.-\left(2 j_{1}+1\right)^{-1} \sum_{n=3}^{N}\left(D_{\underline{j}, \alpha}^{\widetilde{L}_{1}^{n}} \gamma+\left(j_{n}-l_{n}\right) D_{\underline{j}, \alpha}^{\widetilde{L}_{1}} \gamma\right)\right) & \text { for } l_{1}=1
\end{array}
$$

removes the singularity in (6.11). Now if $\gamma$ is horizontal with respect to $\nabla_{\zeta}$ around $\zeta=0$, we have

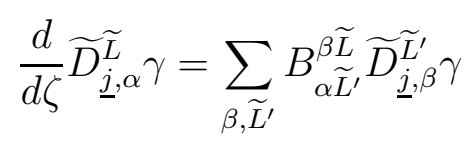

with $B_{\alpha L^{\prime}}^{\beta \widetilde{L}}$ analytic at $\zeta=0$. Consequently, we can extend $\widetilde{D_{j}^{L}, \alpha} \gamma(\zeta)$ to $\zeta=0$. 
Let us introduce

$$
\begin{aligned}
\widetilde{\mathcal{Q}}_{\underline{j}, 2} & =\mathrm{e}^{2 \pi i k(u+\tau / 4)} \mathrm{e}^{\frac{\tau}{2} \partial_{u}} \exp \left[-i z_{2}\left(t_{(1)}^{3}+t_{(2)}^{3}\right)-i z_{3} t_{(3)}^{3}-\cdots-i z_{N} t_{(N)}^{3}\right] \\
& =\mathcal{Q}_{\underline{j}, 2}+\mathcal{O}\left(\zeta^{2(k+2)}\right)
\end{aligned}
$$

with $\varphi_{\zeta} \widetilde{\mathcal{Q}}_{\underline{j}, 2}=\left(\mathcal{Q}_{j^{+}, 2} \oplus \mathcal{Q}_{\underline{j}^{-}, 2}\right) \varphi_{\zeta}$. In terms of states $\widetilde{\gamma}_{j^{ \pm}}$of $\left(\right.$6.6) and for $\alpha=\left(a_{1}+\tau a_{2}\right) / 2, a_{i}=$ 0,1 , operators $\widetilde{D_{\underline{j}, \alpha}^{L}}$ are

$$
\begin{aligned}
\widetilde{D}_{\underline{j}, \alpha}^{\widetilde{L}} \gamma= & \widetilde{D}_{\underline{j}, 0}^{\widetilde{L}} \mathcal{Q}_{\underline{j}, 1}^{a_{1}} \widetilde{\mathcal{Q}}_{\underline{j}, 2}^{a_{2}} \gamma+\mathcal{O}\left(\zeta^{2\left(k+1-2 j_{2}\right)}\right) \\
= & \left(2 j_{2}+1\right) ! D_{\underline{j}^{+}, \alpha}^{\widetilde{L}} \widetilde{\gamma}_{j^{+}} \\
& +\left(2 j_{1}\right) ! \zeta^{2(k+2)} D_{\underline{\underline{j}}^{+}, \alpha}^{\underline{L}} \partial_{v^{\prime}} \widetilde{\gamma}_{j^{+}} \\
& +\left(2 j_{1}-1\right) \zeta^{2\left(k+1-2 j_{2}\right)} D_{\underline{\tilde{j}}^{-}, \alpha} \widetilde{\gamma}_{\underline{j}^{-}} \\
& +\mathcal{O}\left(\zeta^{2\left(k+1-2 j_{2}\right)}\right)
\end{aligned}
$$

and

$$
\begin{aligned}
\widetilde{D}_{\underline{j}, \alpha}^{\widetilde{L}} \gamma= & \widetilde{D}_{\underline{j}, 0}^{\widetilde{L}} \mathcal{Q}_{\underline{j}, 1}^{a_{1}} \widetilde{\mathcal{Q}}_{\underline{j}, 2}^{a_{2}} \gamma+\mathcal{O}\left(\zeta^{2(k+2)}\right) \\
= & \left(2 j_{2}-1\right) ! D_{\underline{j}^{-}, \alpha} \widetilde{\gamma}_{\underline{j}^{-}}+\left(2 j_{1}\right) ! \zeta^{2\left(2 j_{2}+1\right)} D_{\underline{j}^{+}, \alpha}^{\widetilde{L}_{1}} \partial_{v^{\prime}} \widetilde{\gamma}_{\underline{j}^{+}} \\
& +\left(2 j_{1}+1\right)^{-1} \zeta^{2\left(2 j_{2}+1\right)} \sum_{n=3}^{N}\left(\widetilde{D}_{\underline{j}, \alpha}^{L_{1}^{n}} \gamma-\left(j_{n}-l_{n}\right) \widetilde{D}_{\underline{j}, \alpha}^{L_{1}} \gamma\right) \\
& +\mathcal{O}\left(\zeta^{2(k+2)}\right)
\end{aligned}
$$

But equation (6.13) implies that $\widetilde{D}_{\underline{j}, \alpha}^{\widetilde{L}} \gamma=0$ for any $|\widetilde{L}|<J$ and at any value of $\zeta$. For $\zeta=0$ we obtain:

$$
\begin{array}{cl}
D_{\underline{j}^{+}, \alpha}^{L} \widetilde{\gamma}_{\underline{j}^{+}}=0 \quad \text { for } \quad & |L|<j_{2}+1 / 2+j_{3}+\cdots+j_{N} \\
& \alpha=0,1 / 2, \tau / 2,(\tau+1) / 2
\end{array}
$$

and

$$
\begin{array}{ll}
D_{\underline{j}^{-}, \alpha}^{L} \widetilde{\gamma}_{j^{-}}=0 \quad \text { for } \quad & |L|<j_{2}-1 / 2+j_{3}+\cdots+j_{N}, \\
& \alpha=0,1 / 2, \tau / 2,(\tau+1) / 2
\end{array}
$$

which are precisely conditions for bundles $W_{\underline{j}^{ \pm}}^{f r}$. This proves that

$$
\widetilde{W}_{\underline{j}}^{f r}(0)=W_{\underline{j}^{+}}^{f r}\left(\left(z_{2}, \cdots, z_{N}\right), \tau\right) \oplus W_{\underline{j}^{-}}^{f r}\left(\left(z_{2}, \cdots, z_{N}\right), \tau\right) .
$$

Denoting $\mathcal{D}_{j_{1} \cdots j_{N}}=\operatorname{dim} W_{\underline{j}}^{f r}$ we thus obtain

$$
\mathcal{D}_{1 / 2 j_{2} j_{3} \cdots j_{N}}=\mathcal{D}_{\left(j_{2}+1 / 2\right) j_{3} \cdots j_{N}}+\mathcal{D}_{\left(j_{2}-1 / 2\right) j_{3} \cdots j_{N}}
$$


The formula of [31] 26] that we want to prove is

$$
\mathcal{D}_{j_{1} \cdots j_{N}}=\sum_{j=0}^{k / 2} S_{j_{1} j} \cdots S_{j_{N} j} /\left(S_{0 j}\right)^{N}
$$

with

$$
S_{j j^{\prime}}=(2 /(k+2))^{1 / 2} \sin \left[\pi(2 j+1)\left(2 j^{\prime}+1\right) /(k+2)\right] .
$$

Eq. (6.20) is a consequence of (4.15) and of the formal factorization property

$$
\mathcal{D}_{j_{1} \cdots j_{N}}=\sum_{j=0}^{k / 2} N_{j_{1} \cdots j_{m} j} \mathcal{D}_{j j_{m+1} \cdots j_{N}},
$$

where $N_{j_{1} \cdots j_{N}}$ is the dimension of the space of Chern-Simons states in the spherical topology, see [17].

Now, having under control the factorization when one of the spins is $1 / 2$, we can, by an inductive procedure, prove (6.20) rigorously, modulo the absence of toroidal states with spins $>k / 2$. First,

$$
N_{1 / 2 j_{2} j}= \begin{cases}1 & \text { for }\left|j_{2}-j\right|=1 / 2 \text { and } j_{2}, j \leq k / 2 \\ 0 & \text { otherwise }\end{cases}
$$

Formula (6.19) is a particular case of (6.21) and applying it iteratively we can show (6.20) for $j_{2}=\cdots=j_{N}=1 / 2$. For the other cases, we may use an inductive procedure, in the increasing order of the sums $J$ of inserted spins and, for equal $J$ 's, in the decreasing order of the numbers of insertions. Take $j_{1}>1 / 2$. Then, from (6.19),

$$
\mathcal{D}_{j_{1} j_{2} \cdots j_{N}}=\mathcal{D}_{1 / 2\left(j_{1}-1 / 2\right) j_{2} \cdots j_{N}}-\mathcal{D}_{\left(j_{1}-1\right) j_{2} \cdots j_{N}}
$$

and on the right-hand side only earlier sequences appear so that we may proceed inductively. This proves formula (6.20) for the dimension of spaces $W_{\underline{j}}^{f r}$ of $S U(2)$ Chern-Simons states on the torus.

\section{Conclusions}

Let us summarize the main results of the paper. First we have built a stratification of the space of rank two, topologically trivial, holomorphic bundles on the torus. This has been a preliminary step in the description of the states of the $S U(2)$ CS theory in this geometry as polynomials with theta-functions of degree $2 k$ as coefficients. We have constructed the toroidal Knizhnik-Zamolodchikov connection and have shown that the parallel transport preserves the spaces of states. Modulo the integrability condition (if one of spins $>k / 2$ then there are no non-trivial states), we have proven the factorization property of the spaces of states which implies the Verlinde formulae for the dimension of the space of toroidal conformal blocks.

As was remarked before, the integrability condition should follow from the definition of the space of states as it does in the spherical topology or for the toroidal one-point blocks. We 
expect to derive it for toroidal $N$-point blocks by extending the bundles of states to the point where the torus degenerates into the sphere (i.e. when $\tau$ goes to $i \infty$ ). We will address this problem in the future.

It is also interesting to understand the scalar product turning the space of Chern-Simons states into a Hilbert space. In the context of the conformal field theory, the scalar product of states gives the pairing of holomorphic and antiholomorphic conformal blocks into correlation functions. It should be determined uniquely up to normalization by demanding its invariance under the parallel transport with respect to the Knizhnik-Zamolodchikov connection. Up to now we know expressions for the scalar product at genus zero and one in terms of multiple integrals (see [15] 10] for the spherical case). We expect the integrals to converge if (and only

if) we apply them to Chern-Simons states. The proof of this conjecture (still absent for the spherical topology, too) should be the goal of the future research.

\section{Appendix A}

In this Appendix we complete the proof of the stratification of Section 3. We refer the reader to that Section for the notations used here. We shall show that the map of (3.12)

$$
\begin{aligned}
P_{0}: \mathcal{G}_{0}^{\mathbb{C}} \times U_{0} & \longrightarrow \mathcal{A}^{01} \\
\left(g, A_{M}^{01}\right) & \longmapsto{ }^{g} A_{M}^{01}
\end{aligned}
$$

is smooth and injective, its differential is invertible everywhere and its inverse is $C^{\infty}$ when considered as a map

$$
\left(\mathcal{G}_{0}^{\mathbb{C}} \times U_{0}\right) \times \mathcal{A}^{01} \longrightarrow T\left(\mathcal{G}_{0}^{\mathbb{C}} \times U_{0}\right)
$$

Then, by the Nash-Moser Theorem [20], $P_{0}$ is a $C^{\infty}$ diffeomorphism in the Fréchet sense from $\mathcal{G}_{0}^{\mathbb{C}} \times U_{0}$ onto its open image.

It is clear that $P_{0}$ is smooth and tame in the $C^{\infty}$ Fréchet structure of $\mathcal{G}_{0}^{\mathbb{C}} \times U_{0}$ and $\mathcal{A}^{01}$. To prove that $P_{0}$ is injective one has to consider the equation

$$
{ }^{h} A_{M^{\prime}}^{01}=A_{M}^{01} \quad \text { for } h \in \mathcal{G}_{0}^{\mathbb{C}} \text { and } A_{M}^{01}, A_{M^{\prime}}^{01} \in U_{0}
$$

where $A_{M}^{01}, A_{M^{\prime}}^{01}$ are like in (3.10). The general solution of (A.2) is

$$
h(z, \bar{z})=\exp \left(i \frac{z-\bar{z}}{\tau-\bar{\tau}} M\right) g(z) \exp \left(-i \frac{z-\bar{z}}{\tau-\bar{\tau}} M^{\prime}\right)
$$

with $g: \mathbb{C} \rightarrow S L(2, \mathbb{C})$ holomorphic in the complex plane and

$$
\begin{aligned}
g(z+2 \pi) & =g(z) \\
g(z+2 \pi \tau) & =\exp (-2 \pi i M) g(z) \exp \left(2 \pi i M^{\prime}\right) .
\end{aligned}
$$


Upon expanding $g$ in Fourier modes,

$$
g(z)=\sum_{r \in \mathbb{Z}} g_{r} \exp (2 \pi i r z)
$$

eq. (A.4,b) becomes equivalent to the statement that the $2 \times 2$-matrices $g_{r}$ are eigenvectors of

$$
g_{r} \stackrel{\chi}{\mapsto} \exp (-2 \pi i M) g_{r} \exp \left(2 \pi i M^{\prime}\right)
$$

with eigenvalues $\exp (2 \pi i r \tau)$. But eigenvalues of $\chi$ are $\exp \left[ \pm 2 \pi i\left(\operatorname{det}^{1 / 2} M \pm \operatorname{det}^{1 / 2} M^{\prime}\right)\right]$ and, provided (3.11) is satisfied, the only non trivial solution comes from $\operatorname{det} M=\operatorname{det} M^{\prime}$ and $r=0$. This, together with $h(0)=1$, shows that the only solution of (A.2) is $h=1$ and, consequently, that $P_{0}$ is injective.

Now we prove that the differential $D P_{0}$ is invertible and its inverse is a smooth tame map. First note that, with the use of left invariant vector fields to describe vectors in $T \mathcal{G}_{0}^{\mathbb{C}}$,

$$
D P_{0}\left(\left(g, A_{M}^{01}\right)\right)(\phi, B)=g\left(-\bar{\partial} \phi-\left[A_{M}^{01}, \phi\right]+B\right) g^{-1} \equiv g \psi g^{-1} .
$$

Using the expansion in modes

$$
\psi(z, \bar{z})=\sum_{p, r \in \mathbb{Z}} \psi_{p, r} \exp [i(r(z-\bar{z})+p(\bar{\tau} z-\tau \bar{z})) /(\tau-\bar{\tau})]
$$

and the same for $\phi(z, \bar{z})$, one obtains

$$
\begin{aligned}
\psi_{p, r} & =i \frac{r+p \tau}{\tau-\bar{\tau}} \phi_{p, r} d \bar{z}-\left[A_{M}^{01}, \phi_{p, r}\right] \quad \text { for }(p, r) \neq(0,0) \\
\phi_{0,0} & =-\sum_{(p, r) \neq(0,0)} \phi_{p, r} \\
B & =\psi_{0,0}+\left[A_{M}^{01}, \phi_{0,0}\right] .
\end{aligned}
$$

It is evident that this equations can be solved in $\phi$ and $B$ if (A.6,a) can, and this is so if $A_{M}^{01} \in U_{0}$. One easily sees, in this case, that the inverse is a smooth tame family of linear maps, as stated before. We conclude that $P_{0}$ is a $C^{\infty}$ diffeomorphism of Fréchet manifolds.

\section{Appendix B}

Let us explain the passage from eq. (5.9) to (5.11).

The last term inside $(\ldots)$ in $(5.9)$, with the $\sim(z-w)^{-2}$ singularity subtracted, gives the last two terms proportional to $k /(k+2)$ in $[\ldots]$ on the right hand side of (5.11).

The second and the third term in $(\ldots)$ contribute to the third term in $[\ldots]$ the part with $\sum_{r=1}^{\infty}$. 
The rest of (5.11) comes from the first term in $(\ldots)$ of (5.9). The main input is the covariance of Green functions:

$$
\left\langle\Phi_{1} \ldots \Phi_{N}\right\rangle_{\operatorname{Ad}_{\rho} \eta_{u}, 0}=\otimes_{n} D_{R_{n}}(\rho)\left\langle\Phi_{1} \ldots \Phi_{N}\right\rangle_{\eta_{u}, 0} \otimes_{n} D_{R_{n}}\left(\rho^{*}\right)
$$

Then, since $\mathrm{e}^{\epsilon t^{ \pm}} \eta_{u}=\operatorname{Ad}_{\rho(\epsilon)} \eta_{u}+\mathcal{O}\left(\epsilon^{2}\right)$ where $\rho(\epsilon)=\epsilon(1-\exp [ \pm 4 \pi i u])^{-1} t^{ \pm}$

$$
\mathcal{L}_{t^{ \pm}}\left\langle\Phi_{1} \ldots \Phi_{N}\right\rangle_{\eta_{u}, 0}=\sum_{n}(1-\exp [ \pm 4 \pi i u])^{-1} t_{(n)}^{ \pm}\left\langle\Phi_{1} \ldots \Phi_{N}\right\rangle_{\eta_{u}, 0}
$$

Similarly one shows that

$\frac{1}{2} \mathcal{L}_{t^{ \pm}} \mathcal{L}_{t^{\mp}}=\mp(1-\exp [ \pm 4 \pi i u])^{-1} \mathcal{L}_{t^{3}}+\frac{1}{2}\left(\sum_{n}(1-\exp [ \pm 4 \pi i u])^{-1} t_{(n)}^{ \pm}\right)\left(\sum_{n}(1-\exp [\mp 4 \pi i u])^{-1} t_{(n)}^{\mp}\right)$

when acting on $\left\langle\Phi_{1} \ldots \Phi_{N}\right\rangle_{\eta_{u}, 0}$. Finally,

$$
\begin{aligned}
& \frac{1}{2} \mathcal{L}_{t^{ \pm}} \omega\left(z_{n}, w \mid \eta_{u}\right) t_{(n)}^{\mp}
\end{aligned}
$$

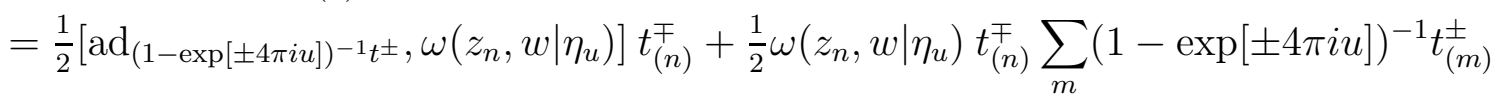

$$
\begin{aligned}
& =\frac{1}{2} \sum_{m}(1-\exp [ \pm 4 \pi i u])^{-1} t_{(m)}^{ \pm} \omega\left(z_{n}, w \mid \eta_{u}\right) t_{(n)}^{\mp}-\frac{1}{2} \omega\left(z_{n}, w \mid \eta_{u}\right) \operatorname{ad}_{(1-\exp [ \pm 4 \pi i u])^{-1} t^{ \pm}} t_{(n)}^{\mp} \\
& =\frac{1}{2} \sum_{m}(1-\exp [ \pm 4 \pi i u])^{-1} t_{(m)}^{ \pm} \omega\left(z_{n}, w \mid \eta_{u}\right) t_{(n)}^{\mp} \mp(1-\exp [ \pm 4 \pi i u])^{-1} \omega\left(z_{n}, w \mid \eta_{u}\right) t_{(n)}^{3} \text {. }
\end{aligned}
$$

\section{Appendix C}

We will list here, without proofs, some elementary facts about the behavior of functions $\omega$ and $\Pi(u, \tau)$ under products and modular transformations. They are useful in studying the connection $\widetilde{\nabla}(w)$. First let introduce some notations:

$$
\begin{aligned}
& \omega^{ \pm}(z, w \mid u)=\operatorname{tr}\left[t ^ { \mp } \left(\omega\left(z, w \mid \eta_{u}\right)+\frac{i}{\left.\left.1-\mathrm{e}^{ \pm 4 \pi i u}\right) t^{ \pm}\right]}\right.\right. \\
& \widetilde{\omega}^{ \pm}(z, w \mid u)=\operatorname{tr}\left[t^{\mp}\left(\widetilde{\omega}\left(z, w \mid \eta_{u}\right)+\frac{i \mathrm{e}^{ \pm 4 \pi i u}}{\left(1-\mathrm{e}^{ \pm 4 \pi i u}\right)^{2}}\right) t^{ \pm}\right], \\
& \widetilde{\omega}(z, w)=\widetilde{\omega}\left(z, w \mid \eta_{u}=1\right) .
\end{aligned}
$$

One has

$$
\begin{aligned}
\omega^{-}\left(z_{n}, w \mid u\right) \omega^{+}\left(z_{m}, w \mid u\right) & =\omega\left(z_{n}, w\right) \omega^{+}\left(z_{m}, z_{n} \mid u\right) \\
& +\omega\left(z_{m}, w\right) \omega^{-}\left(z_{n}, z_{m} \mid u\right)-i \widetilde{\omega}^{+}\left(z_{m}, z_{n} \mid u\right) \text { for } z_{m} \neq z_{n}
\end{aligned}
$$




$$
-i \widetilde{\omega}\left(z_{m}, z_{n}\right)-i \widetilde{\omega}\left(z_{m}, w\right)-i \widetilde{\omega}\left(z_{n}, w\right) \quad \text { for } z_{m} \neq z_{n}
$$

The above formulae are (approximate) versions of the identity

$$
\bar{\partial}^{-1}\left(f \bar{\partial}^{-1} g\right)+\bar{\partial}^{-1}\left(\left(\bar{\partial}^{-1} f\right) g\right)=\left(\bar{\partial}^{-1} f\right)\left(\bar{\partial}^{-1} g\right)
$$

which does not hold exactly because boundary conditions of $\omega$ are not preserved by the products. On the other hand, one has

$$
\begin{gathered}
\omega^{+}\left(z_{n}, w \mid u\right) \omega^{-}\left(z_{n}, w \mid u\right)=\partial_{w} \omega\left(z_{n}, w\right)-i \widetilde{\omega}^{+}\left(z_{n}, z_{n} \mid u\right), \\
\omega\left(z_{n}, w\right) \omega\left(z_{n}, w\right)=\partial_{w} \omega\left(z_{n}, w\right)-i \omega\left(z_{n}, w\right)-i \widetilde{\omega}\left(z_{n}, z_{n}\right)+2 \widetilde{\omega}\left(z_{n}, w\right) .
\end{gathered}
$$

Under shifts and reflections in $u$, quantity $\Pi(u, \tau)$ of eq. (5.19) transforms by

$$
\begin{aligned}
& \Pi(u+1 / 2, \tau)=-\Pi(u, \tau), \\
& \Pi(u+\tau / 2, \tau)=-\mathrm{e}^{-\pi i(4 u+\tau)} \Pi(u, \tau), \\
& \Pi(-u, \tau)=-\Pi(u, \tau)
\end{aligned}
$$

and

$$
\begin{aligned}
& \omega^{ \pm}(z, w \mid-u) \quad=-\omega^{ \pm}(w, z \mid u)=\omega^{\mp}(z, w \mid u), \\
& \omega^{ \pm}(z, w \mid u+1 / 2)=\omega^{ \pm}(z, w \mid u), \\
& \omega^{ \pm}(z, w \mid u+\tau / 2)=\mathrm{e}^{i(z-w)} \omega^{ \pm}(z, w \mid u) .
\end{aligned}
$$

Under the transformations by $\left(\left(\begin{array}{ll}a & b \\ c & d\end{array}\right),\left(p_{n}, q_{n}\right)\right) \in \Gamma_{N}$ of (4.6),

$$
\tau \rightarrow(a \tau+b) /(c \tau+d), \quad z_{n} \rightarrow\left(z_{n}+2 \pi p_{n}+2 \pi \tau q_{n}\right) /(c \tau+d), \quad u \rightarrow u /(c \tau+d),
$$

$\omega$ and $\Pi$ transform as follows

$$
\begin{aligned}
\omega^{ \pm}\left(z_{n}, z_{m} \mid u\right) & \rightarrow(c \tau+d) \omega^{ \pm}\left(z_{n}, z_{m} \mid u\right) \mathrm{e}^{ \pm 2 i u\left[2 \pi\left(q_{n}-q_{m}\right)-c\left(z_{n}-z_{m}\right) /(c \tau+d)\right]} \\
\omega^{3}\left(z_{n}, z_{m}\right) & \rightarrow(c \tau+d)\left(\omega^{3}\left(z_{n}, z_{m}\right)-i\left(q_{n}-q_{m}\right)+i \frac{c\left(z_{n}-z_{m}\right)}{2 \pi(c \tau+d)}+i / 2\right)-i / 2, \\
\Pi(u, \tau) & \rightarrow \pm(c \tau+d)^{1 / 2} \mathrm{e}^{4 \pi i c u^{2} /(c \tau+d)} \Pi(u, \tau) .
\end{aligned}
$$

The last expression is not uniquely defined because of the presence of a square root. This is, however, all we need since $\Pi(u, \tau)$ appears in $\widetilde{\nabla}(w)$ together with its inverse.

\section{Appendix D}


In this Appendix we show how operators $D_{\underline{j}, \alpha}^{L}$ commute with connection $\widetilde{\nabla}(w)$ on bundle $W_{\underline{j}}$. This computation allows to complete the proof of invariance of $W_{\underline{j}}^{f r}(\underline{z}, \tau)$ under parallel transport by the connection.

First we need some simple properties of this operators. Note that generators of the Lie algebra in the polynomial realization are

$$
\begin{aligned}
& t_{(n)}^{3}=-v_{n} \partial_{v_{n}}+j_{n}, \\
& t_{(n)}^{+}=-\partial_{v_{n}} \\
& t_{(n)}^{-}=v_{n}^{2} \partial_{v_{n}}+2 j_{n} v_{n} .
\end{aligned}
$$

Then from the fact that $\sum_{n} t_{(n)}^{3} \gamma=0$ for $\gamma \in W_{\underline{j}}(\underline{z}, \tau)$ one deduces that

$$
\sum_{n=1}^{N} D_{\underline{j}, \alpha}^{L^{n}}=\sum_{n=1}^{N}\left(j_{n}-l_{n}\right) D_{\underline{j}, \alpha}^{L}
$$

( $L^{n}$, resp. $L_{n}$, stands for the $N$-tuple which has all elements equal to those of $L$ except for the $n^{\text {th }}$ one that is increased, resp. decreased, by 1$)$. On the other hand using $(4.2, d)$ one has, for $|L|-J \in 2 \mathbb{Z}+1$

$$
2 D_{\underline{j}, \alpha}^{L}+\sum_{n} l_{n}\left(l_{n}-1-2 j_{n}\right) D_{\underline{j}, \alpha}^{L_{n}}=B_{L^{\prime}}^{L} D_{\underline{j}, \alpha}^{L^{\prime}}
$$

where $B_{L^{\prime}}^{L} \neq 0$ only if $\left|L^{\prime}\right| \leq|L|-2$.

Now consider operators $R_{n}$ of (5.30). Due to (D.2)

$$
\begin{aligned}
\sum_{m} t_{(m)}^{+} \gamma(0) & =\sum_{\substack{L ; \\
l_{0}=0,|L|=J-1}}\left(\prod_{m} v^{l_{m}} / l_{m} !\right) D_{\underline{j}, 0}^{L} \gamma \\
\sum_{m} t_{(m)}^{-} \gamma(0) & =(-1)^{J} \sum_{\substack{L ; \\
l_{0}=0,|L|=J-1}}\left(\prod_{m} v^{2 j_{m}-l_{m}} / l_{m} !\right) D_{\underline{j}, 0}^{L} \gamma
\end{aligned}
$$

and finally for $R_{0}$

$$
\left(\left[\sum_{m} t_{m}^{+}, \sum_{m} t_{m}^{-}\right]-4\right) \partial_{u} \gamma(0)=\sum_{\substack{L, L^{\prime} \\ l_{0}=0 ;|L|=J}}\left(\prod_{m} v^{l^{m}} / l_{m} !\right) B_{L^{\prime}}^{L^{0}} D_{\underline{j}, 0}^{L^{\prime}} \gamma
$$

where $B$ comes from (D.3) i.e. only $\left|L^{\prime}\right|<J$ will give non vanishing terms. This shows that operators $R_{n}$ are zero for $\gamma \in W_{\underline{j}}^{f r}$ as was stated before.

For the rest of the connection we have

$$
\begin{aligned}
D_{\underline{j}, \alpha}^{L} \widetilde{\nabla}(w) \gamma & =(k+2)\left(\sum_{m} \omega\left(z_{m}, w\right) \partial_{z_{m}}+\frac{1}{2 \pi i} \partial_{\tau}\right) D_{\underline{j}, \alpha}^{L} \gamma \\
& +\frac{1}{4 \pi l_{0}} \sum_{m, n} \partial_{u} \operatorname{tr}\left(t^{-} \omega\left(z_{m}, w \mid \eta_{u}\right) t^{+}\right)\left(-2 D_{\underline{j}, \alpha}^{L^{n m}}+2\left(j_{n}-l_{n}\right) D_{\underline{j}, \alpha}^{l^{m}}\right.
\end{aligned}
$$




$$
\begin{aligned}
& \left.-2 \delta_{m n} D_{\underline{j}, \alpha}^{L^{m}}-2\left(l_{m}-j_{m}\right) D_{\underline{j}, \alpha}^{l^{n}}\right) \gamma \\
& -\frac{l_{0}+2}{4 \pi\left(l_{0}+1\right)} \sum_{m} \omega\left(z_{m}, w\right)\left(2 D_{\underline{j}, \alpha}^{L^{0 m}}+2\left(l_{m}-j_{m}\right) D_{\underline{j}, \alpha}^{L^{0}}\right) \gamma \\
& -\frac{1}{4 \pi\left(l_{0}+1\right)} \sum_{m, n} \omega\left(z_{m}, w\right)\left(2\left(l_{n}-j_{n}\right) D_{\underline{j}, \alpha}^{L^{0 m}}-2\left(l_{m}-j_{m}\right) D_{\underline{j}, \alpha}^{L^{0 n}}\right. \\
& +\frac{l_{0}+3}{(4 \pi)^{2}\left(l_{0}+1\right)} D_{\underline{j}, \alpha}^{L^{00}} \gamma-\frac{1}{12(4 \pi)^{2}} \sum_{m, n}\left(D_{\underline{j}, \alpha}^{L^{m n}}+2\left(l_{m}-j_{m}+\delta_{m n}\right) D_{\underline{j}, \alpha}^{L^{n}}\right) \gamma \\
& +\frac{1}{(4 \pi)^{2}\left(l_{0}+1\right)\left(l_{0}+2\right)} \sum_{n, m}\left(D_{\underline{j}, \alpha}^{L^{00 m n}}+2\left(l_{m}-j_{m}\right) D_{\underline{j}, \alpha}^{L^{00 n}}+l_{m}\left(l_{m}-1-2 j_{m}\right) D_{\underline{j}, \alpha}^{L_{m}^{00 n}}\right) \gamma \\
& +\frac{1}{(4 \pi)^{2}\left(l_{0}+1\right)\left(l_{0}+2\right)} \sum_{m}\left(2 D_{\underline{j}, \alpha}^{L^{00 m}}+2\left(l_{m}-j_{m}\right) D_{\underline{j}, \alpha}^{L^{00}}\right) \gamma \\
& +\sum_{\beta, L^{\prime}} A_{\alpha L^{\prime}}^{\beta L} D_{\underline{j}, \beta}^{L^{\prime}} \gamma
\end{aligned}
$$

where $\alpha, \beta=0, \frac{1}{2}, \frac{\tau}{2}, \frac{\tau+1}{2}$ and here and below $A$ represents different matrices with non-zero matrix elements only for $\left|L^{\prime}\right| \leq \max (|L|, J-1)$.

With the application of eq. (D.2), previous expression reduces to

$$
\begin{aligned}
D_{\underline{j}, \alpha}^{L} \nabla(w) \gamma= & (k+2)\left(\sum_{m} \omega\left(z_{m}, w\right) \partial_{z_{m}}+\frac{1}{2 \pi i} \partial_{\tau}\right) D_{\underline{j}, \alpha}^{L} \gamma \\
& +\frac{1}{4 \pi\left(l_{0}+1\right)} \sum_{m} \omega\left(z_{m}, w\right)\left[(J-|L|-2)\left(2 D_{\underline{j}, \alpha}^{L^{0 m}}+2\left(l_{m}-j_{m}\right) D_{\underline{j}, \alpha}^{L^{0}}\right)\right. \\
& \left.\quad-\sum_{n} l_{n}\left(l_{n}-1-2 j_{n}\right) D_{\underline{j}, \alpha}^{L_{n}^{0 m}}\right] \gamma \\
& +\frac{1}{(4 \pi)^{2}\left(l_{0}+1\right)\left(l_{0}+2\right)}\left[(J-|L|-2)\left(|L|-J-2 l_{0}-3\right) D_{\underline{j}, \alpha}^{L^{00}}\right. \\
& \left.+\left(J-|L|+l_{0}+1\right) \sum_{m} l_{m}\left(l_{m}-1-2 j_{m}\right) D_{\underline{j}, \alpha}^{L_{m}^{00}}\right] \gamma \\
& +A_{\alpha L^{\prime}}^{\beta L} D_{\underline{j}, \beta}^{L^{\prime}} \gamma .
\end{aligned}
$$

Note that for $|L| \leq J-2$ eq. (D.7) is equivalent to (5.32). We must still proof that the latter also holds for $|L|=J-1$. In this case, or more generally for $|L|-J+1 \in 2 \mathbb{Z}$, property (D.3) applies and one has

$$
\begin{aligned}
& D_{\underline{j}, \alpha}^{L} \nabla(w) \gamma=(k+2)\left(\sum_{m} \omega\left(z_{m}, w\right) \partial_{z_{m}}+\frac{1}{2 \pi i} \partial_{\tau}\right) D_{\underline{j}, \alpha}^{L} \gamma \\
& \quad+\frac{|L|-J+1}{4 \pi\left(l_{0}+1\right)}\left(\sum_{m} \omega\left(z_{m}, w\right) \sum_{n} l_{n}\left(l_{n}-1-2 j_{n}\right) D_{\underline{j}, \alpha}^{L_{n}^{0 m}}\right. \\
& \left.\quad-\frac{J-|L|+2 l_{0}+4}{4 \pi\left(l_{0}+2\right)} \sum_{n} l_{n}\left(l_{n}-1-2 j_{n}\right) D_{\underline{j}, \alpha}^{L_{n}^{00}}\right) \gamma \\
& \quad+A_{\alpha L^{\prime}}^{\beta L} D_{\underline{j}, \beta}^{L^{\prime}} \gamma .
\end{aligned}
$$

Writing (D.7) and (D.8) in components, one obtains eqs. (5.32). 


\section{References}

[1] Atiyah, M. F., Bott, R.: The Yang-Mills equation over Riemann surfaces. Phil. Trans. R. Soc. Lond. A308, 523-165 (1982)

[2] Axelrod, S., Della Pietra, S., Witten, E.: Geometric quantization of Chern-Simons gauge theory. J. Differential Geometry 33, 787-902 (1991)

[3] Bar-Natan, D., Witten, E.: Perturbative expansion of Chern-Simons theory with noncompact gauge group. Commun. Math. Phys. 141, 423-440 (1991)

[4] Bernard, D.: On the Wess-Zumino-Witten models on the torus. Nucl. Phys. B303, 77-93 (1988)

[5] Bernard, D.: On the Wess-Zumino-Witten models on Riemann surfaces. Nucl. Phys. B309, 145-174 (1988)

[6] Bertram, A., Szenes, A.: Hilbert polynomials of moduli spaces of rank 2 vector bundles II. Harvard University preprint (1991)

[7] Cotta-Ramusino, P., Guadagnini, E., Martellini, M., Mintchev, M.: Quantum field theory and link invariants, Nucl. Phys. B330, 557-574 (1990)

[8] Donaldson, S.: A new proof of a theorem of Narasimhan and Seshadri. J. Diff. Geom. 18, 269-277 (1987)

[9] Elitzur, S., Moore, G., Schwimmer, A., Seiberg, N.: Remarks on the canonical quantization of the Chern-Simons-Witten theory. Nucl. Phys. B326, 104-134 (1989)

[10] Falceto, F., Gawędzki, K., Kupiainen, A.: Scalar product of current blocks in WZW theory. Phys. Lett. B260, 101-108 (1991)

[11] Faltings., G.: Seminar at IHES, July 1992.

[12] Felder, G., Gawędzki, K., Kupiainen, A.: Spectra of Wess-Zumino-Witten with arbitrary simple groups. Commun. Math. Phys. 117 127-158 (1988)

[13] Friedan, D., Shenker, S.: The analytic geometry of two-dimensional conformal field theory. Nucl. Phys. B281, 509-545 (1987)

[14] Gawędzki, K.: Wess-Zumino-Witten conformal field theories. In: Constructive quantum field theory II. Wightman, A. S., Velo, G. (eds.). New York: Plenum 1990, pp. 89-120

[15] Gawędzki, K.: Quadrature of conformal field theories. Nucl. Phys. B328, 733-752 (1989)

[16] Gawędzki, K.: Constructive conformal field theory. In: Functional Integration, Geometry and Strings, eds. Z. Haba et J. Sobczyk, Birkhäuser, Basel, Boston, Berlin 1989, pp. $277-302$ 
[17] Gawȩdzki, K., Kupiainen, A.: SU(2) Chern-Simons theory at genus zero. Commun. Math. Phys. 135, 531-546 (1991)

[18] Guadagnini, E., Martellini, M., Mintchev, M.: Wilson lines in Chern-Simons theory and link invariants, Nucl. Phys. B330, 575-607 (1990)

[19] Gunning, R. C.: Lectures on vector bundles over Riemann surfaces. Princeton: Princeton University Press (1967)

[20] Hamilton, R.: The inverse function theorem of Nash and Moser. Bull. Am. Math. Soc. 7, 65-122 (1982)

[21] Hitchin, N.: Flat connections and geometric quantization. Commun. Math. Phys. 131, 347-380 (1990)

[22] Jeffrey, L. C.: Chern-Simons-Witten invariants of lens spaces and torus bundles, and the semiclassical approximation. Commun. Math. Phys. 147, 563-604 (1992)

[23] Kohno, T.: Linear representations of braid groups and classical Yang-Baxter equations. Contemp. Math. 78, 339-363 (1988)

[24] Knizhnik, V., Zamolodchikov, A. B.: Current algebra and Wess-Zumino model in two dimensions. Nucl. Phys. B247, 83-103 (1984)

[25] Mehta, V., Seshadri, C.: Moduli of vector bundles on curves with parabolic structures. Math. Ann. 248, 205-239 (1980)

[26] Moore, G., Seiberg, N.: Classical and quantum conformal field theory. Commun. Math. Phys. 123, 177-254 (1988)

[27] Narasimhan, M., Seshadri, C.: Stable and unitary vector bundles on a compact Riemann surface. Ann. Math. 82, 540-567 (1965)

[28] Sugawara, H.: A field theory of currents. Phys. Rev. 170, 1659-1662 (1968)

[29] Tsuchiya, A., Kanie, Y., Vertex operators in the conformal field theory on P1 and monodromy representations of the braid group. Adv. Stud. Pure Math. 16, 297-372 (1988)

[30] Tsuchiya, A., Ueno., Y., Yamada, Y.: Conformal field theory on universal family of stable curves with gauge symmetries. Adv. Stud. Pure Math. 19, 459-566 (1989)

[31] Verlinde, E.: Fusion rules and modular transformations in 2-d conformal field theory. Nucl. Phys. B300, 360-376 (1988)

[32] Witten., E.: Non-abelian bosonization in two dimensions. Commun. Math. Phys. 92, 455-472 (1984)

[33] Witten, E.: Quantum field theory and the Jones polynomials. Commun. Math. Phys. 121, 351-399 (1989) 